\title{
Hopf algebra of building sets
}

\author{
Vladimir Grujić* \\ Faculty of Mathematics \\ Belgrade University \\ Serbia \\ vgrujic@matf.bg.ac.rs
}

\author{
Tanja Stojadinović \\ Faculty of Mathematics \\ Belgrade University \\ Serbia \\ tanjas@matf.bg.ac.rs
}

Submitted: Jun 5, 2012; Accepted: Nov 30, 2012; Published: Dec 13, 2012

Mathematics Subject Classifications: 16T05, 16T30, 05E05, 06A11

\begin{abstract}
The combinatorial Hopf algebra on building sets BSet extends the chromatic Hopf algebra of simple graphs. The image of a building set under canonical morphism to quasi-symmetric functions is the chromatic symmetric function of the corresponding hypergraph. By passing from graphs to building sets, we construct a sequence of symmetric functions associated to a graph. From the generalized DehnSommerville relations for the Hopf algebra BSet, we define a class of building sets called eulerian and show that eulerian building sets satisfy Bayer-Billera relations. We show the existence of the cd-index, the polynomial in two noncommutative variables associated to an eulerian building set. The complete characterization of eulerian building sets is given in terms of combinatorics of intersection posets of antichains of finite sets.
\end{abstract}

Keywords: Hopf algebra, building set, graph, symmetric function, DehnSommerville relations, cd-index, simplicial complex

\section{Introduction}

Many combinatorial objects may be endowed with a Hopf algebra structure. The best known examples are Rota's Hopf algebra of finite graded posets [9] and the chromatic Hopf algebra of simple graphs [17].

The theory of combinatorial Hopf algebras is developed in [2]. In Section 2 we recall the basic definitions and properties of combinatorial Hopf algebras and of quasi-symmetric functions. The Hopf algebra of quasi-symmetric functions QSym is the terminal object in

\footnotetext{
*Authors are supported by Ministry of Science of Republic of Serbia, project 174034 .
} 
the category of combinatorial Hopf algebras. It explains the ubiquity of quasi-symmetric functions as generating functions in enumerative combinatorics.

The notion of a building set is originated in the work of De Concini and Procesi [6] in the context of subspace arrangement and developed by Feichtner and Sturmfels [8] and Postnikov [10]. The concept of a building set appears as a combinatorial condition that polytopes from the certain class, called nestohedra, are simple. An example of a building set is provided by the collection of the vertex sets of connected subgraphs in a given graph. Building sets are a kind of Whitney systems. The Hopf algebra on Whitney systems is constructed in [18]. In Section 3, based on Schmitt's work, we introduce the Hopf algebra of building sets $B S e t$ that extends the chromatic Hopf algebra $\mathcal{G}$ of simple graphs.

In Section 4 we define the chromatic symmetric function of a building set as the image under the canonical morphism from building sets to quasi-symmetric functions. To a building set $\mathcal{B}=\mathcal{B}(\mathcal{C})$ is uniquely associated the collection $\mathcal{C}_{\text {min }}$ of minimal elements of the generating collection $\mathcal{C}$, which is an antichain of finite sets. The building sets may be seen as hypergraphs and colorings of building sets are equivalent to colorings of hypergraphs. The chromatic symmetric function of a building set $\mathcal{B}(\mathcal{C})$ depends only on the associated hypergraph $\mathcal{C}_{\text {min }}$. In the setting of building sets, we use the expansion of the chromatic symmetric function of a hypergraph in the basis of the power sum symmetric functions, given in [13]. The derived formulas for the induced chromatic polynomial of a building set are analogues to the classical Whitney's formulas for the chromatic polynomial of a graph $[20]$.

In Section 5 we construct a sequence of algebra morphisms $\beta_{n}$ from graphs to building sets, that produces a sequence of symmetric functions associated to graphs. Two numerical invariants of graphs, the numbers of acyclic and of totally cyclic orientations, arises from this construction.

Every combinatorial Hopf algebra $(\mathcal{H}, \zeta)$ possesses the canonical odd Hopf subalgebra $S_{-}(\mathcal{H}, \zeta)$ on which the character $\zeta$ is odd. This subalgebra is characterized by certain canonical relations, called the generalized Dehn-Sommerville relations. In the case of $\mathcal{H}=Q$ Sym , the generalized Dehn-Sommerville relations are precisely the Bayer-Billera relations for flag $f$-vectors of eulerian posets [3]. In Section 6, by analogy with eulerian posets, we use the generalized Dehn-Sommerville relations for the Hopf algebra BSet to characterize a class of building sets called eulerian. There is no analogues notion of eulerian graphs, because no particular graph satisfies the generalized Dehn-Sommerville relations for the chromatic Hopf algebra of graphs. We derive that eulerian building sets satisfy the Bayer-Billera relations.

The cd- index of an eulerian poset is a polynomial in noncommutative variables, firstly introduced by Fine (see [4]). Its existence is equivalent to the Bayer-Billera relations for the flag $f$-vector. The cd-index is defined for elements of the eulerian subalgebra of an infinitesimal Hopf algebra by a construction given in [1]. In Section 7, by analogy with eulerian posets, we construct the cd-index of eulerian building sets.

In Section 8, we consider how the algebraic condition of being eulerian is related to combinatorics of antichains of finite sets. We obtain the complete characterization and show that eulerian building sets corresponds to clique complexes of chordal graphs. 


\section{Combinatorial Hopf algebras}

In this section we recall the basic definitions and properties of combinatorial Hopf algebras, developed in [2]. Throughout, $n$ is a non-negative integer, $[n]$ denotes the set $\{1, \ldots, n\}$ and $|X|$ denotes the cardinality of a finite set $X$. A composition $\alpha=n$ is a sequence $\alpha=\left(a_{1}, \ldots, a_{k}\right)$ of positive integers with $a_{1}+\cdots+a_{k}=n$. A partition $\lambda \vdash n$ is a multiset $\lambda=\left\{l_{1}, \ldots, l_{k}\right\}$ such that $l_{1}+\cdots+l_{k}=n$.

A combinatorial Hopf algebra $(\mathcal{H}, \zeta)$ is a graded connected Hopf algebra $\mathcal{H}$ over a field $\mathbb{K}$ equipped with a multiplicative linear functional $\zeta: \mathcal{H} \rightarrow \mathbb{K}$, called character. A morphism of combinatorial Hopf algebras $\left(\mathcal{H}_{1}, \zeta_{1}\right)$ and $\left(\mathcal{H}_{2}, \zeta_{2}\right)$ is a morphism of graded Hopf algebras $\phi: \mathcal{H}_{1} \rightarrow \mathcal{H}_{2}$ such that $\zeta_{2} \circ \phi=\zeta_{1}$.

Characters Let $\mathbb{X}(\mathcal{H})$ be the set of characters of an arbitrary Hopf algebra $\mathcal{H}$. The set $\mathbb{X}(\mathcal{H})$, under the convolution product

$$
\varphi \psi=m_{\mathbb{K}} \circ(\varphi \otimes \psi) \circ \Delta_{\mathcal{H}},
$$

is a group with the unit $\epsilon_{\mathcal{H}}$ and the inverse $\varphi^{-1}=\varphi \circ S_{\mathcal{H}}$, where $\epsilon_{\mathcal{H}}$ and $S_{\mathcal{H}}$ are the counit and the antipode of the Hopf algebra $\mathcal{H}$.

Let $\mathcal{H}_{n}$ be the homogeneous component of the grading $n$ of a graded Hopf algebra $\mathcal{H}$. Denote by $\varphi_{n}=\left.\varphi\right|_{\mathcal{H}_{n}}$ the restriction of a character $\varphi$ on the component $\mathcal{H}_{n}$. The conjugate character $\bar{\varphi}$ is defined on homogeneous elements by $\bar{\varphi}(h)=(-1)^{n} \varphi(h), h \in \mathcal{H}_{n}$. A character $\varphi$ is said to be even if $\varphi=\bar{\varphi}$ and it is said to be odd if $\varphi^{-1}=\bar{\varphi}$. Every character $\varphi$ on a graded connected Hopf algebra decomposes uniquely as a product of characters $\varphi=\varphi_{+} \varphi_{-}$, with $\varphi_{+}$even and $\varphi_{-}$odd $([2]$, Theorem 1.5.).

The odd subalgebra $S_{-}(\mathcal{H}, \zeta)$ of a combinatorial Hopf algebra $(\mathcal{H}, \zeta)$ is defined as the largest graded subcoalgebra on which the character $\zeta$ is odd. If $\phi:\left(\mathcal{H}_{1}, \zeta_{1}\right) \rightarrow\left(\mathcal{H}_{2}, \zeta_{2}\right)$ is a morphism of combinatorial Hopf algebras then

$$
\phi\left(S_{-}\left(\mathcal{H}_{1}, \zeta_{1}\right)\right) \subset S_{-}\left(\mathcal{H}_{2}, \zeta_{2}\right) .
$$

For a character $\varphi$ and a composition $\alpha=\left(a_{1}, \ldots, a_{k}\right) \models n$, denote by $\varphi_{\alpha}$ the convolution product

$$
\varphi_{a_{1}} \cdots \varphi_{a_{k}}: \mathcal{H} \stackrel{\Delta(k-1)}{\longrightarrow} \mathcal{H}^{\otimes k} \stackrel{\text { proj }}{\longrightarrow} \mathcal{H}_{a_{1}} \otimes \cdots \otimes \mathcal{H}_{a_{k}} \stackrel{\varphi^{\otimes k}}{\longrightarrow} \mathbb{K}
$$

Quasi-symmetric functions The basic reference for quasi-symmetric functions is [11]. The algebra QSym of quasi-symmetric functions is a graded subalgebra of the algebra $\mathbb{K}\left[\left[x_{1}, x_{2}, \ldots\right]\right]$ of formal power series of finite degree in countably variables. It is linearly spanned by monomial quasi-symmetric functions

$$
M_{\alpha}=\sum_{i_{1}<i_{2}<\cdots<i_{k}} x_{i_{1}}^{a_{1}} x_{i_{2}}^{a_{2}} \cdots x_{i_{k}}^{a_{k}}
$$


where $\alpha=\left(a_{1}, a_{2}, \ldots a_{k}\right) \models n$ is a composition of an integer $n \in \mathbb{N}$. It is a graded Hopf algebra with coproduct

$$
\Delta\left(M_{\alpha}\right)=\sum_{\alpha=\beta \gamma} M_{\beta} \otimes M_{\gamma}
$$

where $\beta \gamma$ is the concatenation of compositions $\beta$ and $\gamma$.

Let $\zeta: \mathbb{K}\left[\left[x_{1}, x_{2}, \ldots\right]\right] \rightarrow \mathbb{K}$ be an algebra morphism defined on variables by $\zeta\left(x_{1}\right)=1$ and $\zeta\left(x_{i}\right)=0$ for $i \neq 1$. The universal character $\zeta_{Q}$ on $Q S y m$ is the restriction $\zeta_{Q}=$ $\left.\zeta\right|_{\text {QSym }}$. It is determined on the monomial basis by

$$
\zeta_{Q}\left(M_{\alpha}\right)=\left\{\begin{array}{lc}
1, & \alpha=(n) \text { or }() \\
0, & \text { otherwise }
\end{array} .\right.
$$

One of the main results of ([2], Theorem 4.1.) is that for an arbitrary combinatorial Hopf algebra $(\mathcal{H}, \zeta)$, there is a unique morphism of combinatorial Hopf algebras $\Psi:(\mathcal{H}, \zeta) \longrightarrow$ $\left(Q S y m, \zeta_{Q}\right)$, which is defined on homogeneous elements $h \in \mathcal{H}_{n}$ by

$$
\Psi(h)=\sum_{\alpha \models n} \zeta_{\alpha}(h) M_{\alpha}
$$

The morphism $\Psi$ we call the canonical morphism of the combinatorial Hopf algebra $(\mathcal{H}, \zeta)$.

Given a composition $\alpha=\left(a_{1}, \ldots, a_{k}\right) \models n$, let $s(\alpha)=\left\{a_{1}, \ldots, a_{k}\right\} \vdash n$ be the corresponding partition. The Hopf algebra of symmetric functions $S y m$ is a Hopf subalgebra of $Q S y m$ linearly spanned by monomial symmetric functions $m_{\lambda}=\sum_{s(\alpha)=\lambda} M_{\alpha}$, where $\lambda$ runs over all partitions. The principal specialization of symmetric functions is an assignment of the value at $x_{1}=x_{2}=\cdots=x_{m}=1, x_{m+1}=x_{m+2}=\cdots=0$ to a symmetric function $\phi \in$ Sym. It is a polynomial in $m$ denoted by $\phi\left(1^{m}\right)$.

\section{Building sets}

Definition 3.1. A collection $\mathcal{B} \subset \mathcal{P}(X)$ of non-empty subsets of a finite set $X$ is a building set on $X$ if it satisfies two conditions:

(B1) If $S, S^{\prime} \in \mathcal{B}$ and $S \cap S^{\prime} \neq \emptyset$ then $S \cup S^{\prime} \in \mathcal{B}$

(B2) $\{i\} \in \mathcal{B}$ for all $i \in X$.

We write $\mathcal{B}_{X}$ to indicate the ground set $X$. If we do not require the condition (B2), a family $\mathcal{B}$ is called a Whitney system on $X$ [18]. Building sets on $X$ are ordered by inclusion. The minimal building set contains only singletons $\mathcal{D}_{X}=\{\{i\} \mid i \in X\}$. We call $\mathcal{D}_{X}$ the discrete building set on $X$. The maximal building set $\mathcal{P}_{X}=\mathcal{P}(X) \backslash\{\emptyset\}$ contains all non-empty subsets of $X$. The $\operatorname{rank} \operatorname{rank}(\mathcal{B})$ of a building set $\mathcal{B}$ is the cardinality of the ground set $X$. The restriction of a building set $\mathcal{B}$ to a subset $I \subset X$ is a building set on $I$ defined by $\left.\mathcal{B}\right|_{I}=\{S \in \mathcal{B} \mid S \subset I\}$.

Let $\mathcal{B}_{X}$ and $\mathcal{B}_{Y}$ be building sets on finite sets $X$ and $Y$. A map $f: X \longrightarrow Y$ is a map of building sets if $f^{-1}(S) \in \mathcal{B}_{X}$ for all $S \in \mathcal{B}_{Y}$. We say that building sets $\mathcal{B}_{X}$ and $\mathcal{B}_{Y}$ are equivalent if there is a bijection $f: X \longrightarrow Y$ such that $f(S) \in \mathcal{B}_{Y} \Leftrightarrow S \in \mathcal{B}_{X}$. 
The elements of a building set are ordered by inclusion. The restriction $\left.\mathcal{B}\right|_{I}$ to a maximal element $I \in \mathcal{B}$ is called a connected component of $\mathcal{B}$. Every building set is a disjoint union of its connected components. A building set $\mathcal{B}_{X}$ is connected if $X \in \mathcal{B}_{X}$. The minimal connected building set that contains $\mathcal{B}$ is $\overline{\mathcal{B}}=\mathcal{B} \cup\{X\}$.

Suppose that is given an arbitrary collection $\mathcal{C} \subset \mathcal{P}(X)$ of subsets of a finite set $X$, such that every $S \in \mathcal{C}$ has at least two elements. Define inductively the sequence of collections

$$
\mathcal{C}_{0}=\mathcal{C}, \mathcal{C}_{k+1}=\mathcal{C}_{k} \cup\left\{S \cup S^{\prime} \mid S \in \mathcal{C}_{0}, S^{\prime} \in \mathcal{C}_{k}, S \cap S^{\prime} \neq \emptyset\right\}, k \geqslant 0 .
$$

The union $\mathcal{B}(\mathcal{C})=\bigcup_{k \geqslant 0} \mathcal{C}_{k}$, with all singletons $\{x\}, x \in X$ added, is a building set on $X$. For a building set $\mathcal{B}$ on $X$ there is a unique minimal collection $\mathcal{C} \subset \mathcal{P}(X)$ such that $\mathcal{B}=\mathcal{B}(\mathcal{C})$. We call such collection the generating collection of a building set $\mathcal{B}$.

The motivating example of a building set comes from graph theory.

Example 3.2. The graph $\Gamma=(V, E)$, with the sets of vertices $V$ and edges $E$, is called simple if there are no either multiple edges nor loops. For a simple graph $\Gamma=(V, E)$ a collection $\mathcal{B}(\Gamma)=\left\{I \subset V|\Gamma|_{I}\right.$ is connected $\}$ is a building set on $V$. We call $\mathcal{B}(\Gamma)$ the graphical building set corresponding to the graph $\Gamma$. Note that the graphical building set $\mathcal{B}(\Gamma)$ is connected if and only if the graph $\Gamma$ is connected. Also for each subset of vertices $I \subset V$ the restriction $\left.\mathcal{B}(\Gamma)\right|_{I}$ is the graphical building set $\mathcal{B}\left(\left.\Gamma\right|_{I}\right)$ corresponding to the induced subgraph $\left.\Gamma\right|_{I}$. The set of edges $E$ is the generating collection of the graphical building set $\mathcal{B}(\Gamma)$.

Let $B S e t$ be the vector space over the field $\mathbb{K}$ of characteristic zero, spanned by all equivalence classes of building sets and $B S e t_{n}$ its subspace spanned by equivalence classes of building sets of rank $n$. The space $B$ Set, endowed with product $\mathcal{B}_{X} \cdot \mathcal{B}_{Y}=\mathcal{B}_{X} \sqcup \mathcal{B}_{Y}$, where $\mathcal{B}_{X} \sqcup \mathcal{B}_{Y}=\left\{S \subset X \sqcup Y \mid S \in \mathcal{B}_{X}\right.$ or $\left.S \in \mathcal{B}_{Y}\right\}$ is a building set on disjoint union $X \sqcup Y$, and coproduct

$$
\Delta\left(\mathcal{B}_{X}\right)=\left.\left.\sum_{I \subset X}\left(\mathcal{B}_{X}\right)\right|_{I} \otimes\left(\mathcal{B}_{X}\right)\right|_{I^{c}}
$$

is a graded, connected commutative and cocommutative Hopf algebra. The unit is the building set $\mathcal{B}_{\emptyset}$ on the empty set. Denote by Conn the family of all equivalence classes of connected building sets. Then, as the algebra, BSet is isomorphic to the polynomial algebra $\mathbb{K}[$ Conn $]$ generated by the family Conn. As a graded, connected bialgebra, BSet possesses the antipode $S: B S e t \rightarrow B S e t$, determined by

$$
S\left(\mathcal{B}_{X}\right)=\left.\sum_{k \geqslant 1}(-1)^{k} \sum_{J_{1} \sqcup \ldots \sqcup J_{k}=X} \prod_{j=1}^{k}\left(\mathcal{B}_{X}\right)\right|_{J_{j}},
$$

for building sets on $X \neq \emptyset$, where the inner sum is over all ordered k-tuples $\left(J_{1}, \ldots, J_{k}\right)$ of non-empty disjoint subsets, whose union is equal to $X$. 


\section{Chromatic symmetric function of a building set}

Let $\zeta$ be a character on the Hopf algebra of building sets $B S e t$ defined by

$$
\zeta(\mathcal{B})=\left\{\begin{array}{cc}
1, & \mathcal{B} \text { is discrete } \\
0, & \text { otherwise }
\end{array} .\right.
$$

For a building set $\mathcal{B}_{X}$ on the set $X$ with $n$ elements and a composition $\alpha=\left(a_{1}, \ldots, a_{k}\right) \models$ $n$, the value of the convolution product $\zeta_{\alpha}\left(\mathcal{B}_{X}\right)$, defined by $(1)$, is the number of ordered decompositions $X=J_{1} \sqcup \ldots \sqcup J_{k}$ such that $\left.\left(\mathcal{B}_{X}\right)\right|_{J_{i}}$ is discrete and $\left|J_{i}\right|=a_{i}$, for all $i=$ $1, \ldots, k$. We call a function $f: X \rightarrow \mathbb{N}$ a proper coloring of a building set $\mathcal{B}_{X}$ if for every set $S \in \mathcal{B}_{X}$ with at least two elements, there are $i, j \in S$ such that $f(i) \neq f(j)$. For each ordered decomposition $X=J_{1} \sqcup \ldots \sqcup J_{k}$ such that $\left.\left(\mathcal{B}_{X}\right)\right|_{J_{i}}$ is discrete for all $i=1, \ldots k$ and positive integers $n_{1}<\cdots<n_{k}$, there is a proper coloring $f$ given by $\left.f\right|_{J_{i}}=n_{i}$. Conversely, each proper coloring $f: X \rightarrow \mathbb{N}$ of the building set $\mathcal{B}_{X}$, with $f(X)=\left\{n_{1}<\cdots<n_{k}\right\}$, defines an ordered decomposition $X=f^{-1}\left(\left\{n_{1}\right\}\right) \sqcup \ldots f^{-1}\left(\left\{n_{k}\right\}\right)$, where $\left.\left(\mathcal{B}_{X}\right)\right|_{f^{-1}\left(\left\{n_{i}\right\}\right)}$ is discrete for all $i=1, \ldots k$.

Definition 4.1. The chromatic symmetric function of a building set $\mathcal{B}_{X} \in B$ Set ${ }_{n}$ is its image under the canonical morphism $\Psi:($ BSet,$\zeta) \rightarrow\left(Q S y m, \zeta_{Q}\right)$, given by $(2)$ with

$$
\Psi\left(\mathcal{B}_{X}\right)=\sum_{\alpha \models n} \zeta_{\alpha}\left(\mathcal{B}_{X}\right) M_{\alpha}
$$

The function $\Psi\left(\mathcal{B}_{X}\right)$ is obviously symmetric. The principal specialization of the function $\Psi\left(\mathcal{B}_{X}\right)$ counts proper colorings with finite number of colors.

Definition 4.2. The chromatic polynomial $\chi\left(\mathcal{B}_{X}, m\right)$ of a building set $\mathcal{B}_{X}$ is the principal specialization

$$
\chi\left(\mathcal{B}_{X}, m\right)=\Psi\left(\mathcal{B}_{X}\right)\left(1^{m}\right) .
$$

Since the principal specialization on monomial basis is given by $M_{\alpha}\left(1^{m}\right)=\left(\begin{array}{c}m \\ k(\alpha)\end{array}\right)$, where $k(\alpha)$ is the length of a composition $\alpha=n$, we obtain

$$
\chi\left(\mathcal{B}_{X}, m\right)=\sum_{\alpha \models n} \zeta_{\alpha}\left(\mathcal{B}_{X}\right)\left(\begin{array}{c}
m \\
k(\alpha)
\end{array}\right) .
$$

We are especially interested in the value of the chromatic polynomial of a building set at $m=-1$, which is

$$
\chi\left(\mathcal{B}_{X},-1\right)=\sum_{\alpha \models n}(-1)^{k(\alpha)} \zeta_{\alpha}\left(\mathcal{B}_{X}\right)
$$

It defines some numerical invariant, which we call the $(-1)$-invariant of building sets.

Recall the definition of the Hopf algebra of simple graphs, considered by Schmitt, [17]. Let $\mathcal{G}$ be the $\mathbb{K}$-vector space spanned by all equivalence classes of finite simple graphs, 
graded by the number of vertices of a graph. The space $\mathcal{G}$ is a graded, commutative and cocommutative Hopf algebra with product $\Gamma_{1} \cdot \Gamma_{2}=\Gamma_{1} \sqcup \Gamma_{2}$ (the disjoint union of graphs) and coproduct

$$
\Delta(\Gamma)=\left.\left.\sum_{I \subset V} \Gamma\right|_{I} \otimes \Gamma\right|_{I^{c}}
$$

where $V$ is the set of vertices of a graph $\Gamma$ and $\left.\Gamma\right|_{I}$ its restriction on vertices $I \subset V$. Let $\zeta_{\mathcal{G}}: \mathcal{G} \rightarrow \mathbb{K}$ be

$$
\zeta_{\mathcal{G}}(\Gamma)=\left\{\begin{array}{lc}
1, & \Gamma \text { is discrete } \\
0, & \text { otherwise }
\end{array} .\right.
$$

The canonical morphism $\Psi_{\mathcal{G}}:\left(\mathcal{G}, \zeta_{\mathcal{G}}\right) \rightarrow\left(Q S y m, \zeta_{Q}\right)$, given by (2) with

$$
\Psi_{\mathcal{G}}(\Gamma)=\sum_{\alpha \models n}\left(\zeta_{\mathcal{G}}\right)_{\alpha}(\Gamma) M_{\alpha}, \Gamma \in \mathcal{G}_{n}
$$

is Stanley's chromatic symmetric function of a graph, constructed in [12]. Its principal specialization produces the chromatic polynomial $\chi(\Gamma, m)=\Psi_{\mathcal{G}}(\Gamma)\left(1^{m}\right)$ of a graph. Let $\beta: \mathcal{G} \rightarrow B$ Set be the map that sends a graph $\Gamma$ to the corresponding graphical building set $\mathcal{B}(\Gamma)$.

Theorem 4.3. The map $\beta: \mathcal{G} \rightarrow$ BSet is a monomorphism of combinatorial Hopf algebras such that $\Psi \circ \beta=\Psi_{\mathcal{G}}$.

Proof. First we show that $\beta$ is an algebra morphism. Indeed, $\beta\left(\Gamma_{1} \cdot \Gamma_{2}\right)$ is the graphical building set corresponding to the disjoint union $\Gamma_{1} \sqcup \Gamma_{2}$. It contains all subsets $S \subset$ $V\left(\Gamma_{1}\right) \sqcup V\left(\Gamma_{2}\right)$ such that the restriction $\left.\left(\Gamma_{1} \sqcup \Gamma_{2}\right)\right|_{S}$ is connected. It is exactly the product of graphical building sets $\mathcal{B}\left(\Gamma_{1}\right) \cdot \mathcal{B}\left(\Gamma_{2}\right)$. Recall that $\mathcal{B}\left(\left.\Gamma\right|_{I}\right)=\left.\mathcal{B}(\Gamma)\right|_{I}$. Therefore,

$$
\Delta(\beta(\Gamma))=\left.\left.\sum_{I \subset V(\Gamma)} \mathcal{B}(\Gamma)\right|_{I} \otimes \mathcal{B}(\Gamma)\right|_{I^{c}}=\sum_{I \subset V(\Gamma)} \mathcal{B}\left(\left.\Gamma\right|_{I}\right) \otimes \mathcal{B}\left(\left.\Gamma\right|_{I^{c}}\right)=(\beta \otimes \beta)(\Delta(\Gamma)) .
$$

Thus, the map $\beta$ is a coalgebra morphism. Since the graphical building set $\mathcal{B}(\Gamma)$ is discrete if and only if the graph $\Gamma$ is discrete, we have $\zeta \circ \beta=\zeta_{\mathcal{G}}$. The correspondence of graphs and graphical building sets is bijective, so the map $\beta$ is a monomorphism.

It follows from Theorem 4.3 that chromatic polynomials of graphical building sets and chromatic polynomials of graphs coincide

$$
\chi(\mathcal{B}(\Gamma), m)=\chi(\Gamma, m) .
$$

A classical theorem of Stanley asserts that evaluating the chromatic polynomial of a graph at -1 gives the number of acyclic orientation [14]. Therefore the $(-1)$-invariant (4) is a generalization of the number of acyclic orientations to building sets.

An arbitrary collection of subsets $\mathcal{H} \subset \mathcal{P}(X)$ of the ground set $X$ is called a hypergraph on $X$. A proper coloring of a hypergraph $\mathcal{H}$ is a map $f: X \rightarrow \mathbb{N}$, such that for any $S \in \mathcal{H}$ 
with at least two elements, $f$ is not monochromatic on $S$. The chromatic symmetric function of a hypergraph is defined as

$$
\Psi(\mathcal{H})=\sum_{\text {proper } f: X \rightarrow \mathbb{N}} \prod_{i \in X} x_{f(i)}
$$

It depends only on minimal elements of a hypergraph, which form an antichain in the boolean poset $\mathcal{P}(X)$. The specialization

$$
\chi(\mathcal{H}, m)=\Psi(\mathcal{H})\left(1^{m}\right)
$$

is the chromatic polynomial, which counts the number of proper colorings of $\mathcal{H}$ with $m$ colors.

The following theorem, which is a simple consequence of definitions, shows in what extent the chromatic symmetric function distinguishes building sets.

Theorem 4.4. Let $\mathcal{C}_{\text {min }}$ be the collection of minimal elements of the generating collection $\mathcal{C}$ of a building set $\mathcal{B}(\mathcal{C})$. Then

$$
\Psi(\mathcal{B}(\mathcal{C}))=\Psi\left(\mathcal{C}_{\min }\right)
$$

where $\Psi\left(\mathcal{C}_{\text {min }}\right)$ is the chromatic symmetric function of the hypergraph $\mathcal{C}_{\text {min }}$.

Proof. A coloring $f: X \rightarrow \mathbb{N}$ is a proper coloring of $\mathcal{B}(\mathcal{C})$ if and only if $f$ is not monochromatic on an arbitrary $S \in \mathcal{C}_{\text {min }}$. Thus, the building set $\mathcal{B}(\mathcal{C})$ and the hypergraph $\mathcal{C}_{\text {min }}$ have the same sets of proper colorings, so their chromatic symmetric functions are equal.

Define the minimalization of a building set $\mathcal{B}=\mathcal{B}(\mathcal{C})$ as the building set $\check{\mathcal{B}}=\mathcal{B}\left(\mathcal{C}_{\text {min }}\right)$. By Theorem 4.4, building sets $\mathcal{B}\left(\mathcal{C}^{\prime}\right)$ and $\mathcal{B}\left(\mathcal{C}^{\prime \prime}\right)$, with the same minimalizations, have the same chromatic symmetric functions

$$
\Psi\left(\mathcal{B}\left(\mathcal{C}^{\prime}\right)\right)=\Psi\left(\mathcal{B}\left(\mathcal{C}^{\prime \prime}\right)\right)
$$

The fundamental property of chromatic polynomials of graphs is the deletioncontraction property

$$
\chi(\Gamma, m)=\chi(\Gamma \backslash e, m)-\chi(\Gamma / e, m),
$$

where $\Gamma \backslash e$ is $\Gamma$ with an edge $e \in E(\Gamma)$ deleted and $\Gamma / e$ is $\Gamma$ with $e$ contracted to a point. The deletion-contraction recurrence was proved for the chromatic polynomial of a hypergraph in [19]. We state the deletion-contraction property in the setting of building sets. Let $S \in \mathcal{C}_{\text {min }}$ be a minimal element of the generating collection $\mathcal{C}$ of a building set $\mathcal{B}=\mathcal{B}(\mathcal{C})$ on the ground set $X$. The deletion is a building set $\mathcal{B}(\mathcal{C} \backslash\{S\})$ on $X$, generated by the collection $\mathcal{C} \backslash\{S\}$. We denote it by $\mathcal{B} \backslash S$ without confusing with the set-theoretic deletion. Denote by $X / S=X \backslash S \cup\{S\}$. For a subset $A \subset X$ denote by $A / S=\left\{\begin{array}{ll}A, & A \cap S=\emptyset \\ A \backslash S \cup\{S\}, & A \cap S \neq \emptyset\end{array}\right.$, which is a subset of $X / S$. The contraction is a building set $\mathcal{B} / S=\mathcal{B}(\mathcal{C} / S)$ on $X / S$, generated by the collection $\mathcal{C} / S=\{A / S \mid A \in \mathcal{C}\}$. 


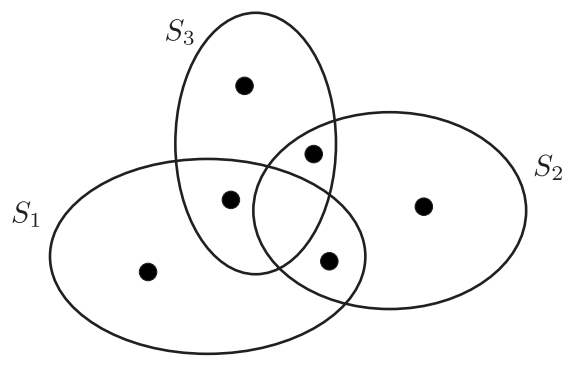

$\mathcal{B}=\mathcal{B}\left(\left\{S_{1}, S_{2}, S_{3}\right\}\right)$

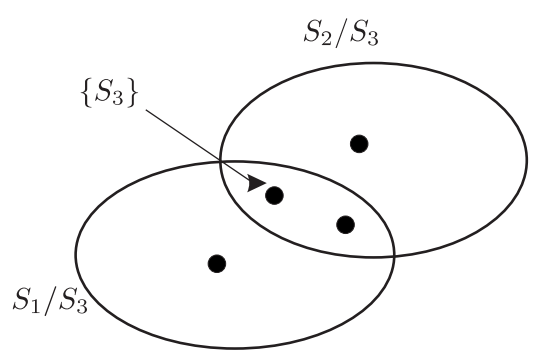

$\mathcal{B} / S_{3}=\mathcal{B}\left(\left\{S_{1} / S_{3}, S_{2} / S_{3}\right\}\right)$

Figure 1: The contraction of a building set

Theorem 4.5. Let $S \in \mathcal{C}_{\min }$ be a minimal element of the generating collection $\mathcal{C}$ of a building set $\mathcal{B}=\mathcal{B}(\mathcal{C})$. Then chromatic polynomials of the building set $\mathcal{B}$ and its deletion $\mathcal{B} \backslash S$ and contraction $\mathcal{B} / S$ are related by

$$
\chi(\mathcal{B}, m)=\chi(\mathcal{B} \backslash S, m)-\chi(\mathcal{B} / S, m) .
$$

Proof. Every proper coloring $f: X \rightarrow[m]$ of $\mathcal{B}$ is a proper coloring of $\mathcal{B} \backslash S$. A proper coloring $f: X \rightarrow[m]$ of $\mathcal{B} \backslash S$ is a proper coloring of $\mathcal{B}$ if and only if $f(i) \neq f(j)$ for some $i, j \in S$. The formula follows from the fact that the set of proper colorings $f: X \rightarrow[m]$ of $\mathcal{B} \backslash S$ which are monochromatic on $S$ and the set of all proper colorings of $\mathcal{B} / S$ have the same number of elements.

Definition 4.6. Let $\mathcal{L}$ be a finite antichain of nonempty finite sets. We say that a set $S \in \mathcal{L}$ is a free set of $\mathcal{L}$ if $S \cap \cup(\mathcal{L} \backslash\{S\})$ has at most one element.

The next proposition is an immediate consequence of the deletion-contraction property of chromatic polynomials of building sets.

Proposition 4.7. Given a building set $\mathcal{B}=\mathcal{B}(\mathcal{C})$ and its minimalization $\check{\mathcal{B}}=\mathcal{B}\left(\mathcal{C}_{\text {min }}\right)$. If $S \in \mathcal{C}_{\min }$ is a free set of $\mathcal{C}_{\min }$ then

(i) $\chi(\mathcal{B}, m)=\chi(\check{\mathcal{B}} \backslash S, m)\left(m^{|S|}-m\right)$ if $S \cap \cup\left(\mathcal{C}_{\min } \backslash\{S\}\right)=\emptyset$

(ii) $\chi(\mathcal{B}, m)=\chi(\check{\mathcal{B}} \backslash S, m)\left(m^{|S|-1}-1\right)$ if $S \cap \cup\left(\mathcal{C}_{\text {min }} \backslash\{S\}\right)$ has one element.

In the following two theorems are given expansions of chromatic symmetric functions of building sets in the power sum basis of symmetric functions. These expansions are just restatements in the setting of building sets of Stanley's expansions of chromatic symmetric functions of hypergraphs [13]. They are analogues to the power sum expansions of chromatic symmetric functions of graphs [12]. The derived formulas for the chromatic polynomial of a building set extend the classical Whitney's formulas for the chromatic polynomial of a graph [20]. 
Theorem 4.8. ([13], Theorem 3.5) Let $\mathcal{C}_{\min }$ be the collection of minimal elements of the generating collection $\mathcal{C}$ of a building set $\mathcal{B}=\mathcal{B}(\mathcal{C})$ on the ground set $X$. For a subcollection $\mathcal{S} \subset \mathcal{C}_{\min }$, let $\lambda(\mathcal{S})$ be the partition of $\operatorname{rank}(\mathcal{B})$ whose parts are equal to the ranks of connected components of the building set $\mathcal{B}(\mathcal{S})$ on $X$. Then

$$
\Psi(\mathcal{B})=\sum_{\mathcal{S} \subset \mathcal{C}_{\min }}(-1)^{|\mathcal{S}|} p_{\lambda(\mathcal{S})} .
$$

The principal specializations of the power sum symmetric functions are given by $p_{\lambda}\left(1^{m}\right)=m^{|\lambda|}$, where $|\lambda|$ is the size of a partition $\lambda$. For a subcollection $\mathcal{S} \subset \mathcal{C}_{\text {min }}$ denote by $c(\mathcal{S})=|\lambda(\mathcal{S})|$, the number of connected components of the building set $\mathcal{B}(\mathcal{S})$ on the ground set $X$. We obtain the following formula for the chromatic polynomials of building sets

$$
\chi(\mathcal{B}, m)=\sum_{\mathcal{S} \subset \mathcal{C}_{\min }}(-1)^{|\mathcal{S}|} m^{c(\mathcal{S})},
$$

which is also a direct consequence of the deletion-contraction property. It gives the following interpretation of the $(-1)$-invariant of building sets

$$
\chi(\mathcal{B},-1)=\sum_{\mathcal{S} \subset \mathcal{C}_{\min }}(-1)^{|\mathcal{S}|+c(\mathcal{S})} .
$$

Let $\mathcal{L}=\left\{S_{1}, \ldots, S_{m}\right\}$ be an antichain of the boolean poset $\mathcal{P}(X)$. Denote by $\mathcal{L}_{I}=$ $\left\{S_{i} \mid i \in I\right\}$ the subcollection of $\mathcal{L}$ determined by a subset $I \subset[\mathrm{m}]$. The intersection poset $P(\mathcal{L})$ of the collection $\mathcal{L}$ is the set $P(\mathcal{L})=\left\{I \subset[m] \mid \cap \mathcal{L}_{I} \neq \emptyset\right\}$ ordered by inclusion.

Proposition 4.9. Let $\mathcal{L}^{\prime}=\left\{S_{1}^{\prime}, \ldots, S_{m}^{\prime}\right\}$ and $\mathcal{L}^{\prime \prime}=\left\{S_{1}^{\prime \prime}, \ldots, S_{m}^{\prime \prime}\right\}$ be antichains of finite sets with the same intersection poset $P\left(\mathcal{L}^{\prime}\right)=P\left(\mathcal{L}^{\prime \prime}\right)$. If $\left|\cap \mathcal{L}_{I}^{\prime}\right|=\left|\cap \mathcal{L}_{I}^{\prime \prime}\right| \bmod 2$ for all $I \in P$ then

$$
\chi\left(\mathcal{B}\left(\mathcal{L}^{\prime}\right),-1\right)=\chi\left(\mathcal{B}\left(\mathcal{L}^{\prime \prime}\right),-1\right) .
$$

Proof. Given an antichain of finite sets $\mathcal{L}=\left\{S_{1}, \ldots, S_{m}\right\}$, denote by $S_{I}=\cap_{i \in I} S_{i}$ and $X_{I}=$ $\cup_{i \in I} S_{i}$ for all $I \subset[m]$. Let $c(I)$ and $c_{I}$ be the numbers of connected components of building sets $\mathcal{B}\left(\mathcal{L}_{I}\right)$ on the ground sets $X_{[m]}$ and $X_{I}$ respectively. Since $c(I)=c_{I}+\left|X_{[m]}\right|-\left|X_{I}\right|$, by inclusion-exclusion, we obtain

$$
c(I)=c_{I}+\left|X_{[m]}\right|+\sum_{J \subset I}(-1)^{|J|}\left|S_{J}\right| .
$$

By formula (5), we have that $\chi(\mathcal{B}(\mathcal{L}),-1)=\sum_{I \subset[m]}(-1)^{|I|+c(I)}$, which depends only on the intersection poset $P(\mathcal{L})$ and the parity of $\left|S_{I}\right|$ for all $I \in P(\mathcal{L})$. 
To a building set $\mathcal{B}=\mathcal{B}(\mathcal{C})$ on the ground set $X$ is associated the lattice $L_{\mathcal{B}}$ of connected partitions of its minimalization $\check{\mathcal{B}}=\mathcal{B}\left(\mathcal{C}_{\text {min }}\right)$. For a partition $\pi=\left\{A_{1}, A_{2}, \ldots, A_{k}\right\}$ of $X$ let type $(\pi)$ be a partition of $|X|$ whose components are sizes of blocks. A partition $\pi$ of $X$ is said to be connected if the restrictions to blocks $\left.\breve{\mathcal{B}}\right|_{A}, A \in \pi$ are connected as building sets. A set of all connected partitions is ordered by refinement of partitions, with the unique minimal element $\widehat{0}$, which is the partition of $X$ in one-element blocks. Denote by $|\pi|$ the number of blocks of a partition $\pi$. Let $\mu$ be the Moebius function of a lattice $L_{\mathcal{B}}$.

Theorem 4.10. ([13], Theorem 3.4) Let $L_{\mathcal{B}}$ be the lattice of connected partitions of the building set $\mathcal{B}\left(\mathcal{C}_{\min }\right)$ associated to a building set $\mathcal{B}=\mathcal{B}(\mathcal{C})$. Then

$$
\Psi(\mathcal{B})=\sum_{\pi \in L_{\mathcal{B}}} \mu(\widehat{0}, \pi) p_{\text {type }(\pi)} .
$$

By the principal specialization of the power sum symmetric functions, we obtain from Theorem 4.10 the following interpretation of the chromatic polynomial and (-1)- invariant of a building set

$$
\begin{gathered}
\chi(\mathcal{B}, m)=\sum_{\pi \in L_{\mathcal{B}}} \mu(\widehat{0}, \pi) m^{|\pi|}, \\
\chi(\mathcal{B},-1)=\sum_{\pi \in L_{\mathcal{B}}} \mu(\widehat{0}, \pi)(-1)^{|\pi|} .
\end{gathered}
$$

\section{Symmetric functions of graphs derived from build- ing sets}

To a simple graph $\Gamma=(V, E)$ and an integer $n \geqslant 2$ we associate a collection of sets $\mathcal{C}_{\Gamma, n}$ in the following way. To an edge $e \in E$ we associate a set of new objects $\left\{e_{1}, \ldots, e_{n-2}\right\}$. Define $S_{e}=\left\{u, v, e_{1}, \ldots, e_{n-2}\right\}$, where $e=\{u, v\} \in E$ is an edge on vertices $u, v \in V$. The collection $\mathcal{C}_{\Gamma, n}=\left\{S_{e} \mid e \in E\right\}$ is an antichain on the ground set $X=V \cup\left\{e_{i} \mid e \in E, i \in\right.$ $[n-2]\}$. It generates uniquely the building set $\mathcal{B}_{\Gamma, n}=\mathcal{B}\left(\mathcal{C}_{\Gamma, n}\right)$ on $X$, see Fig. 2.

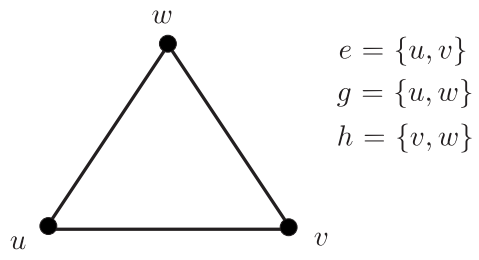

$K_{3}$

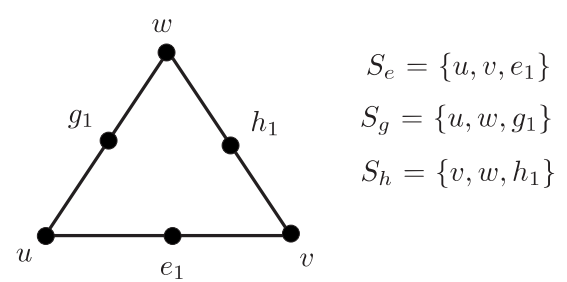

$\beta_{3}\left(K_{3}\right)=\mathcal{B}\left(\left\{S_{e}, S_{g}, S_{h}\right\}\right)$

Figure 2: The building set $\beta_{3}\left(K_{3}\right)$ 
Define a sequence of maps $\beta_{n}: \mathcal{G} \rightarrow B$ Set, $n \geqslant 2$, by $\beta_{n}(\Gamma)=\mathcal{B}_{\Gamma, n}$, for $\Gamma \in \mathcal{G}$. Note that $\beta_{2}$ is the Hopf algebra monomorphism $\beta$ in Theorem 4.3. It is clear from the construction that all $\beta_{n}$ are algebra monomorphisms. To each graph $\Gamma$ we associate chromatic symmetric functions $\Psi\left(\beta_{n}(\Gamma)\right)$ of building sets $\beta_{n}(\Gamma)$ and corresponding chromatic polynomials $\chi\left(\beta_{n}(\Gamma), m\right)$. We obtain a sequence of multiplicative invariants of graphs.

By Proposition 4.9, if $n_{1}=n_{2} \bmod 2$ then $\chi\left(\beta_{n_{1}}(\Gamma),-1\right)=\chi\left(\beta_{n_{2}}(\Gamma),-1\right)$ for any simple graph $\Gamma$. We obtain two numerical multiplicative invariants of graphs, derived from $(-1)$-invariant of building sets, namely $\chi\left(\beta_{2}(\Gamma),-1\right)$ and $\chi\left(\beta_{3}(\Gamma),-1\right)$.

Denote by $c(S)$ the number of connected components of the spanning subgraph $(V, S)$ with edge set $S \subset E$. The correspondence between subsets $S \subset E$ of edges of a graph $\Gamma$ and subcollections $\mathcal{S}=\left\{S_{e} \mid e \in S\right\}$ of the generating collection $\mathcal{C}_{\Gamma, n}$ of the building set $\beta_{n}(\Gamma)$ is bijective. From the construction, we have

$$
c(\mathcal{S})=c(S)+(n-2)(|E|-|S|),
$$

where $c(\mathcal{S})$ is the number of connected components of the building set $\mathcal{B}(\mathcal{S})$. For $n=2,3$ it follows from (5) that

$$
\begin{aligned}
& \chi\left(\beta_{2}(\Gamma),-1\right)=\sum_{S \subset E}(-1)^{|S|+c(S)}, \\
& \chi\left(\beta_{3}(\Gamma),-1\right)=\sum_{S \subset E}(-1)^{|E|+c(S)} .
\end{aligned}
$$

These formulas appears to be evaluations of the Tutte polynomial of a graph $\Gamma$

$$
T_{\Gamma}(x, y)=\sum_{S \subset E}(x-1)^{c(S)-c(E)}(y-1)^{c(S)+|S|-|V|} .
$$

We obtain $\chi\left(\beta_{2}(\Gamma),-1\right)=(-1)^{|V|} T_{\Gamma}(2,0)$ and $\chi\left(\beta_{3}(\Gamma),-1\right)=(-1)^{|E|+c(E)} T_{\Gamma}(0,2)$. The combinatorial interpretation of these invariants is well known. The values $T_{\Gamma}(2,0)$ and $T_{\Gamma}(0,2)$ are the numbers of acyclic and of totally cyclic orientations of $\Gamma$ (see, e.g. [5]). Recall that an oriented graph is acyclic if it contains no directed cycles and that it is totally cyclic if every edge is contained in some directed cycle.

Example 5.1. In [12], Stanley gave the example of nonisomorphic five-vertex graphs that have the same chromatic symmetric function, Fig. 3. By direct calculation we obtain

$$
\Psi\left(\beta_{3}\left(\Gamma_{1}\right)\right)-\Psi\left(\beta_{3}\left(\Gamma_{2}\right)\right)=-p_{5,3,1,1,1}+p_{6,3,1,1}+p_{7,1,1,1,1}-2 p_{8,1,1,1}+2 p_{9,1,1}-p_{10,1} .
$$

The invariant $\chi\left(\beta_{3}(\Gamma),-1\right)$, derived from the chromatic symmetric function $\Psi\left(\beta_{3}(\Gamma)\right)$, distinguishes those graphs.

Remark 5.2. It is natural to ask in what extant chromatic symmetric functions $\Psi\left(\beta_{n}(\Gamma)\right)$ distinguish graphs. The previous example shows that they are at least incomparable with the chromatic symmetric function of a graph. 

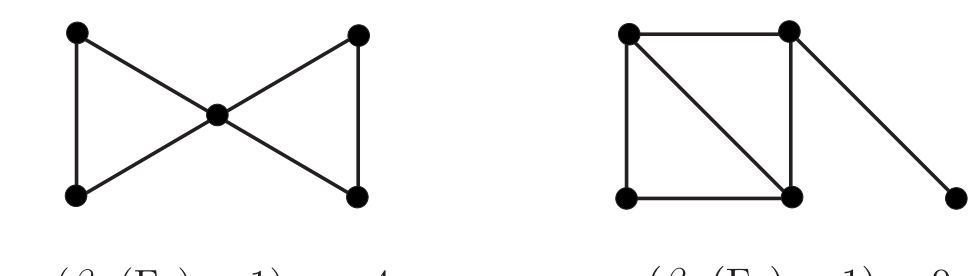

$$
\chi\left(\beta_{3}\left(\Gamma_{1}\right),-1\right)=-4 \quad \chi\left(\beta_{3}\left(\Gamma_{2}\right),-1\right)=0
$$

Figure 3: Stanley's example of graphs with the same chromatic symmetric function

Remark 5.3. The invariant $\chi\left(\beta_{3}(\Gamma),-1\right)$ takes values of both signs on graphs of the same rank, in the range $-2^{|E|}<\chi\left(\beta_{3}(\Gamma),-1\right)<2^{|E|}$. Hence there is not an analogous result to the reciprocity theorem for the chromatic polynomials of graphs, [15].

\section{Generalized Dehn-Sommerville relations for build- ing sets}

Let $S_{-}(\mathcal{H}, \zeta)$ be the odd subalgebra of the combinatorial Hopf algebra $(\mathcal{H}, \zeta)$. It is proved in ([2], Theorem 5.3.) that a homogeneous element $h \in \mathcal{H}$ belongs to $S_{-}(\mathcal{H}, \zeta)$ if and only if

$$
\left(i d \otimes\left(\bar{\zeta}-\zeta^{-1}\right) \otimes i d\right) \circ \Delta^{(2)}(h)=0 .
$$

We refer to the previous equation as the generalized Dehn-Sommerville relations for the combinatorial Hopf algebra $(\mathcal{H}, \zeta)$.

For the Hopf algebra of quasi-symmetric functions $\left(Q S y m, \zeta_{Q}\right)$, these relations are described in ([2], Example 5.10.) as follows. Let $h=\sum_{\alpha \models n} f_{\alpha}(h) M_{\alpha}$ be a homogeneous element of order $n \in \mathbb{N}$. It satisfies the generalized Dehn-Sommerville relations if and only if

$$
\sum_{j=0}^{a_{i}}(-1)^{j} f_{\left(a_{1}, \ldots, a_{i-1}, j, a_{i}-j, a_{i+1}, \ldots, a_{k}\right)}(h)=0,
$$

for each composition $\alpha=\left(a_{1}, \ldots, a_{k}\right) \models n$ and $i \in\{1, \ldots, k(\alpha)\}$. By $k(\alpha)$ is denoted the number of parts of the composition $\alpha$. It is understood that zero parts in compositions are omitted. We refer to the relations (7) as the Bayer-Billera relations [3].

Denote by $\mathcal{H}^{c o p}$ the coopposite Hopf algebra of a Hopf algebra $\mathcal{H}$ (see [7] as the general reference for Hopf algebras). It is defined by the coproduct $\Delta^{c o p}=\tau \circ \Delta$, where $\tau: \mathcal{H} \otimes \mathcal{H} \rightarrow \mathcal{H} \otimes \mathcal{H}$ is the twist map $\tau(x \otimes y)=y \otimes x, x, y \in \mathcal{H}$. Let $\mathcal{H}$ be a commutative Hopf algebra. A map $\phi: \mathcal{H} \rightarrow \mathcal{H}$ is an antimorphism of $\mathcal{H}$ if it is a morphism of Hopf algebras $\phi: \mathcal{H} \rightarrow \mathcal{H}^{\text {cop }}$.

Lemma 6.1. Let $(\mathcal{H}, \zeta)$ be a commutative combinatorial Hopf algebra. Then the antipode $S_{\mathcal{H}}:\left(\mathcal{H}^{c o p}, \zeta^{-1}\right) \rightarrow(\mathcal{H}, \zeta)$ is a morphism of combinatorial Hopf algebras. 
Proof. The antipode $S_{\mathcal{H}}: \mathcal{H} \rightarrow \mathcal{H}$ is an antimorphism of coalgebras. As $\mathcal{H}$ is commutative, we have that $S_{\mathcal{H}}: \mathcal{H}^{\text {cop }} \rightarrow \mathcal{H}$ is a morphism of Hopf algebras. Since the inverse of a character $\zeta$ is the composition $\zeta^{-1}=\zeta \circ S_{\mathcal{H}}$, the claim follows.

Lemma 6.2. Let $S_{Q}$ and $S_{B}$ be antipodes of Hopf algebras QSym and BSet respectively. Then the following diagram is a commuting diagram of morphisms of combinatorial Hopf algebras:

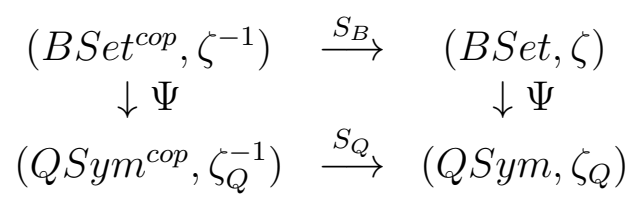

Proof. The map $\Psi$ commutes with antipodes as a morphism of Hopf algebras, so we need only prove that the above maps are morphisms of combinatorial Hopf algebras. The Hopf algebras QSym and BSet are commutative. By Lemma 6.1, we have that both $S_{Q}:\left(Q S y m^{c o p}, \zeta_{Q}^{-1}\right) \rightarrow\left(Q S y m, \zeta_{Q}\right)$ and $S_{B}:\left(B S e t^{c o p}, \zeta^{-1}\right) \rightarrow(B S e t, \zeta)$ are morphisms of combinatorial Hopf algebras. The map $\Psi: B S e t^{c o p} \rightarrow Q S y m^{c o p}$ is a morphism of Hopf algebras. It follows from

$$
\zeta_{Q}^{-1} \circ \Psi=\zeta_{Q} \circ S_{Q} \circ \Psi=\zeta_{Q} \circ \Psi \circ S_{B}=\zeta \circ S_{B}=\zeta^{-1},
$$

that $\Psi:\left(B S e t^{c o p}, \zeta^{-1}\right) \rightarrow\left(Q S y m^{c o p}, \zeta_{Q}^{-1}\right)$ is a morphism of combinatorial Hopf algebras as well.

We obtain the following formula for the inverse of the character $\zeta$ on the Hopf algebra of building sets.

Proposition 6.3. The value $\zeta^{-1}\left(\mathcal{B}_{X}\right)$ is the $(-1)$-invariant of a building set $\mathcal{B}_{X} \in$ BSet $_{n}$

$$
\zeta^{-1}\left(\mathcal{B}_{X}\right)=\chi\left(\mathcal{B}_{X},-1\right) .
$$

Proof. The inverse of the universal character $\zeta_{Q}$ on the Hopf algebra of quasi-symmetric functions QSym, calculated in ([2], Example 4.8.), is given on the monomial basis by

$$
\zeta_{Q}^{-1}\left(M_{\alpha}\right)=(-1)^{k(\alpha)}
$$

Hence, by Lemma 6.2, we have

$$
\zeta^{-1}\left(\mathcal{B}_{X}\right)=\zeta_{Q}^{-1} \circ \Psi\left(\mathcal{B}_{X}\right)=\zeta_{Q}^{-1}\left(\sum_{\alpha \models n} \zeta_{\alpha}\left(\mathcal{B}_{X}\right) M_{\alpha}\right)=\sum_{\alpha \models n}(-1)^{k(\alpha)} \zeta_{\alpha}\left(\mathcal{B}_{X}\right) .
$$

This is precisely, by (4), the $(-1)$-invariant of a building set $\mathcal{B}_{X}$. 
Example 6.4. Let $d_{n}=\zeta^{-1}\left(\mathcal{D}_{n}\right), n \in \mathbb{N}$ be values of $\zeta^{-1}$ on discrete building sets $\mathcal{D}_{n}, n \in \mathbb{N}$. Setting $\mathcal{D}_{n}, n \in \mathbb{N}$ in the identity $\zeta^{-1} * \zeta=\epsilon$ gives the following recursive relation $\sum_{i=0}^{n} d_{i}\left(\begin{array}{c}n \\ i\end{array}\right)=0, n \in \mathbb{N}$, where $d_{0}=\zeta^{-1}\left(\mathcal{B}_{\emptyset}\right)=1$, which is uniquely satisfied by $d_{n}=(-1)^{n}, n \in \mathbb{N}$. The same is obtained by calculating the chromatic polynomial of a discrete building set $\chi\left(\mathcal{D}_{n}, m\right)=m^{n}$ at $m=-1$. Denote by $\left(\begin{array}{l}n \\ \alpha\end{array}\right)=\left(\begin{array}{c}n \\ a_{1} \\ a_{2} \cdots a_{k}\end{array}\right)=\frac{n !}{a_{1} ! a_{2} ! \cdots a_{k} !}$ the multinomial coefficient corresponding to the composition $\alpha=\left(a_{1}, a_{2}, \ldots, a_{k}\right) \models n$. For a discrete building set $\mathcal{D}_{n}$ and a composition $\alpha \models n$, we have $\zeta_{\alpha}\left(\mathcal{D}_{n}\right)=\left(\begin{array}{l}n \\ \alpha\end{array}\right)$. We obtain the following identity

$$
\sum_{\alpha \models n}(-1)^{k(\alpha)}\left(\begin{array}{l}
n \\
\alpha
\end{array}\right)=(-1)^{n} .
$$

By Theorem 4.5, we have that the inverse character $\zeta^{-1}$ satisfies the deletioncontraction property. Let $\mathcal{C}$ be the generating collection of a building set $\mathcal{B}=\mathcal{B}(\mathcal{C})$ and $S \in \mathcal{C}_{\text {min }}$ be a minimal element of collection $\mathcal{C}$. Then

$$
\zeta^{-1}(\mathcal{B})=\zeta^{-1}(\mathcal{B} \backslash S)-\zeta^{-1}(\mathcal{B} / S)
$$

The following lemma is an immediate consequence of Proposition 4.7.

Lemma 6.5. Let $\mathcal{B}=\mathcal{B}(\mathcal{C})$ be a building set such that the minimal collection $\mathcal{C}_{\text {min }}$ contains a free set of odd cardinality. Then $\zeta^{-1}(\mathcal{B})=0$.

The conjugate character $\bar{\zeta}$ on $B$ Set is given by

$$
\bar{\zeta}\left(\mathcal{B}_{X}\right)=\left\{\begin{array}{cc}
(-1)^{n}, & \mathcal{B}_{X} \text { is discrete of rank } n \\
0, & \text { otherwise }
\end{array}\right.
$$

According to the generalized Dehn-Sommerville relations (6) for the combinatorial Hopf algebra of building sets $(B S e t, \zeta)$, we have

$$
\mathcal{B}_{X} \in S_{-}(B S e t, \zeta) \text { if and only if }\left(i d \otimes\left(\zeta^{-1}-\bar{\zeta}\right) \otimes i d\right) \circ \Delta^{(2)}\left(\mathcal{B}_{X}\right)=0 .
$$

It follows from

$$
\begin{gathered}
\left(i d \otimes\left(\zeta^{-1}-\bar{\zeta}\right) \otimes i d\right) \circ \Delta^{(2)}\left(\mathcal{B}_{X}\right)=\left.\left.\sum_{I \sqcup J \sqcup K=X}\left(\mathcal{B}_{X}\right)\right|_{I} \otimes\left(\zeta^{-1}-\bar{\zeta}\right)\left(\left.\left(\mathcal{B}_{X}\right)\right|_{J}\right) \otimes\left(\mathcal{B}_{X}\right)\right|_{K}= \\
\sum_{J \subset X}\left(\zeta^{-1}-\bar{\zeta}\right)\left(\left.\left(\mathcal{B}_{X}\right)\right|_{J}\right) \Delta\left(\left.\left(\mathcal{B}_{X}\right)\right|_{J^{c}}\right) \text {, that } \\
\mathcal{B}_{X} \in S_{-}(B S e t, \zeta) \text { if }\left(\zeta^{-1}-\bar{\zeta}\right)\left(\left.\left(\mathcal{B}_{X}\right)\right|_{J}\right)=0 \text { for all } J \subset X .
\end{gathered}
$$

Definition 6.6. A building set $\mathcal{B}_{X}$ is an eulerian building set if for all subsets $J \subset X$, either $\left.\left(\mathcal{B}_{X}\right)\right|_{J}$ is discrete or $\zeta^{-1}\left(\left.\left(\mathcal{B}_{X}\right)\right|_{J}\right)=0$. 
By Proposition 6.3 and formula (5), the property of being eulerian for the building set $\mathcal{B}=\mathcal{B}(\mathcal{C})$ depends only on the collection of minimal elements $\mathcal{C}_{\text {min }}$, i.e $\mathcal{B}$ is eulerian if and only if its minimalization $\check{\mathcal{B}}=\mathcal{B}\left(\mathcal{C}_{\text {min }}\right)$ is eulerian. From (9) and (10) we obtain the following property.

Proposition 6.7. If $\mathcal{B}_{X}$ is an eulerian building set then $\mathcal{B}_{X} \in S_{-}(B S e t)$.

It follows from the definition that for any eulerian building set $\mathcal{B}_{X}$ and a subset $J \subset X$, the restriction $\left.\left(\mathcal{B}_{X}\right)\right|_{J}$ is an eulerian building set as well. Let $\mathcal{E}$ Set be the subspace of $B$ Set spanned by all eulerian building sets. Restrictions of an eulerian building set and disjoint unions of eulerian building sets are again eulerian, so the subspace $\mathcal{E}$ Set is a Hopf subalgebra of the Hopf algebra of building sets BSet.

Example 6.8. Let $\overline{\mathcal{D}}_{n}=\{\{1\},\{2\}, \ldots,\{n\},[n]\}$. From (8) we obtain $\zeta^{-1}\left(\overline{\mathcal{D}}_{n}\right)=(-1)^{n}+$ 1. The same is a simple consequence of the deletion-contraction property. Thus, $\overline{\mathcal{D}}_{n}$ is eulerian if and only if $n$ is odd. Let $\mathcal{C}$ be the generating collection of an eulerian building set $\mathcal{B}=\mathcal{B}(\mathcal{C})$ and $S \in \mathcal{C}_{\text {min }}$ be a minimal element of the collection $\mathcal{C}$. The restriction $\left.\mathcal{B}\right|_{S}=\overline{\mathcal{D}}_{|S|}$ is eulerian, so $S$ has an odd number of elements. Specially, nondiscrete graphical building sets are not eulerian. It means that there is no analogues notion of eulerian graphs in the combinatorial Hopf algebra $\left(\mathcal{G}, \zeta_{\mathcal{G}}\right)$.

Theorem 6.9. Let $\mathcal{B}_{X}$ be an eulerian building set. Then

$$
\sum_{j=0}^{a_{i}}(-1)^{j} \zeta_{\left(a_{1}, \ldots, a_{i-1}, j, a_{i}-j, a_{i+1}, \ldots, a_{k}\right)}\left(\mathcal{B}_{X}\right)=0
$$

where $\alpha=\left(a_{1}, \ldots, a_{k}\right) \models|X|$ and $i \in\{1, \ldots, k(\alpha)\}$.

Proof. Let $\Psi:(B S e t, \zeta) \longrightarrow\left(Q S y m, \zeta_{Q}\right)$ be the canonical morphism of the Hopf algebra $B$ Set. Since

$$
\mathcal{E} \text { Set } \subset S_{-}(B \text { Set }, \zeta)
$$

and

$$
\Psi\left(S_{-}(B S e t, \zeta)\right) \subset S_{-}\left(Q S y m, \zeta_{Q}\right),
$$

relations (11) follow from the Bayer-Billera relations (7).

\section{The cd-index of eulerian building sets}

The $\mathbf{c d}-$ index $\Phi_{P}(\mathbf{c}, \mathbf{d})$ of an eulerian poset $P$ is a polynomial in noncommutative variables, which efficiently encodes the flag $f$-vector. Its existence is equivalent to the Bayer-Billera relations ([4], Theorem 4.). There is a general result for the existence of the cd-index which is known from [1]. The algebra of noncommutative polynomials $\mathbb{K}<\mathbf{a}, \mathbf{b}\rangle$ with the comultiplication defined by $\Delta(\mathbf{a})=\Delta(\mathbf{b})=1 \otimes 1$ is the terminal object in the category of infinitesimal Hopf algebras. The canonical morphism sends the 
eulerian subalgebra of an infinitesimal Hopf algebra to the subalgebra of polynomials generated by variables $\mathbf{c}=\mathbf{a}+\mathbf{b}$ and $\mathbf{d}=\mathbf{a b}+\mathbf{b a}$. In this section we construct the $\mathbf{c d}$-index of eulerian building sets directly, by analogy with Stanley's proof of the existence of the cd-index of eulerian posets ([16], Theorem 1.1).

To a composition $\alpha=\left(a_{1}, a_{2}, \ldots, a_{k}\right) \models n$ is associated the set $S(\alpha)=\left\{a_{1}, a_{1}+\right.$ $\left.a_{2}, \ldots, a_{1}+\cdots+a_{k-1}\right\} \subset[n-1]$. The compositions are ordered by $\beta \preceq \alpha$ if and only if $S(\beta) \subset S(\alpha)$. Let $u_{\alpha}$ be a monomial in two noncommutative variables $\mathbf{a}$ and $\mathbf{b}$ defined by

$$
u_{\alpha}=\prod_{i=1, n} u_{\alpha, i}, \text { where } u_{\alpha, i}=\left\{\begin{array}{ll}
\mathbf{a}, & i \notin S(\alpha) \\
\mathbf{b}, & i \in S(\alpha)
\end{array} .\right.
$$

Given a building set $\mathcal{B}$ of the rank $n$. Define the flag $f$-vector of $\mathcal{B}$ to be $\left(\zeta_{\alpha}(\mathcal{B})\right)_{\alpha \models n}$ and the flag $h$-vector $\left(\eta_{\alpha}(\mathcal{B})\right)_{\alpha \models n}$ to be a regular linear transformation

$$
\eta_{\alpha}(\mathcal{B})=\sum_{\beta \preceq \alpha}(-1)^{k(\alpha)-k(\beta)} \zeta_{\beta}(\mathcal{B}) .
$$

The $\mathbf{a b}$-index of $\mathcal{B}$ is the generating function of the flag $h$-vector

$$
\Psi_{\mathcal{B}}(\mathbf{a}, \mathbf{b})=\sum_{\alpha \models n} \eta_{\alpha}(\mathcal{B}) u_{\alpha}
$$

Theorem 7.1. If $\mathcal{B} \in \mathcal{E}$ Set is an eulerian building set then there is a polynomial $\Phi_{\mathcal{B}}(\mathbf{c}, \mathbf{d})$ in variables $\mathbf{c}=\mathbf{a}+\mathbf{b}$ and $\mathbf{d}=\mathbf{a b}+\mathbf{b a}$, called the $\mathbf{c d}-$ index of $\mathcal{B}$, such that

$$
\Psi_{\mathcal{B}}(\mathbf{a}, \mathbf{b})=\Phi_{\mathcal{B}}(\mathbf{c}, \mathbf{d})
$$

Proof. Let $\mathcal{B}$ be an eulerian building set of the rank $n$ on the ground set $X$. It is an immediate consequence of (12) that

$$
\Psi_{\mathcal{B}}(\mathbf{a}+\mathbf{b}, \mathbf{b})=\sum_{\alpha \models n} \zeta_{\alpha}(\mathcal{B}) u_{\alpha}
$$

Therefore,

$$
\Psi_{\mathcal{B}}(\mathbf{a}, \mathbf{b})=\sum_{k \geqslant 1} \sum_{S_{1} \sqcup \ldots \sqcup S_{k}=X}(\mathbf{a}-\mathbf{b})^{\left|S_{1}\right|-1} \mathbf{b}(\mathbf{a}-\mathbf{b})^{\left|S_{2}\right|-1} \mathbf{b} \cdots(\mathbf{a}-\mathbf{b})^{\left|S_{k}\right|-1},
$$

where the inner sum is over all ordered decompositions $X=S_{1} \sqcup \ldots \sqcup S_{k}$, such that the restrictions $\left.\mathcal{B}\right|_{S_{i}}, i=1, \ldots, k$ are discrete. Let $I(B S e t)$ be the incidence algebra of $B S e t$ over the ring of noncommutative polynomials $\mathbb{Q}\langle\mathbf{a}, \mathbf{b}\rangle$. Define functionals $f, g, h \in$ $I$ (BSet) by

$$
\begin{gathered}
f(\mathcal{B})=\mathbf{a} \Psi_{\mathcal{B}}(\mathbf{a}, \mathbf{b}), g(\mathcal{B})=\mathbf{b} \Psi_{\mathcal{B}}(\mathbf{a}, \mathbf{b}), \\
h(\mathcal{B})=\left\{\begin{array}{cc}
(\mathbf{a}-\mathbf{b})^{n}, & \mathcal{B} \text { is discrete of rank } n \\
0, & \text { otherwise }
\end{array}\right.
\end{gathered}
$$


From the equation (13) follows

$$
\Psi_{\mathcal{B}}(\mathbf{a}, \mathbf{b})=\sum_{\emptyset \neq I \subset X:\left.\mathcal{B}\right|_{I} \text { discrete }}(\mathbf{a}-\mathbf{b})^{|I|-1} \mathbf{b} \Psi_{\left.\mathcal{B}\right|_{I^{c}}}(\mathbf{a}, \mathbf{b})
$$

which gives $f=h * g$. By the formula (3) for the antipode $S$ of the Hopf algebra BSet, we obtain the inverse of $h$

$$
\begin{gathered}
h^{-1}(\mathcal{B})=h \circ S(\mathcal{B})=h\left(\left.\sum_{k \geqslant 1}(-1)^{k} \sum_{J_{1} \sqcup \ldots \sqcup J_{k}=X} \prod_{i=1, k} \mathcal{B}\right|_{J_{i}}\right)= \\
=(\mathbf{a}-\mathbf{b})^{|X|} \sum_{k \geqslant 1}(-1)^{k} \mid\left\{J_{1} \sqcup \ldots \sqcup J_{k}=X|\mathcal{B}|_{J_{i}} \text { discrete, } i=1, k\right\} \mid=\zeta^{-1}(\mathcal{B})(\mathbf{a}-\mathbf{b})^{|X|} .
\end{gathered}
$$

Hence for eulerian building sets we have

$$
h^{-1}(\mathcal{B})=\left\{\begin{array}{cc}
(-1)^{n}(\mathbf{a}-\mathbf{b})^{n}, & \mathcal{B} \text { is discrete of rank } n \\
0, & \text { otherwise }
\end{array}, \mathcal{B} \in \mathcal{E} \text { Set } .\right.
$$

By definition, the restrictions $\left.\mathcal{B}\right|_{I}, I \subset X$ of an eulerian building set $\mathcal{B}$ satisfy either $\zeta^{-1}\left(\left.\mathcal{B}\right|_{I}\right)=0$ or $\left.\mathcal{B}\right|_{I}$ is discrete. Therefore the identity $g=h^{-1} * f$ gives the following

$$
\Psi_{\mathcal{B}}(\mathbf{a}, \mathbf{b})=\sum_{\emptyset \neq I \subset X:\left.\mathcal{B}\right|_{I}} \text { discrete }(-1)^{|I|-1}(\mathbf{a}-\mathbf{b})^{|I|-1} \mathbf{a} \Psi_{\left.\mathcal{B}\right|_{I^{c}}}(\mathbf{a}, \mathbf{b}) .
$$

Summing up the equations (14) and (15) gives the following recursive formula

$$
\Psi_{\mathcal{B}}(\mathbf{a}, \mathbf{b})=\frac{1}{2} \sum_{|I| \text { odd }}\left(\mathbf{c}^{2}-2 \mathbf{d}\right)^{\frac{|I|-1}{2}} \mathbf{c} \Psi_{\left.\mathcal{B}\right|_{I^{c}}}(\mathbf{a}, \mathbf{b})-\frac{1}{2} \sum_{|I| \text { even }}\left(\mathbf{c}^{2}-2 \mathbf{d}\right)^{\frac{|I|}{2}} \Psi_{\left.\mathcal{B}\right|_{I^{c}}}(\mathbf{a}, \mathbf{b})
$$

where the sums are over all nonempty subsets $I \subset X$ such that $\left.\mathcal{B}\right|_{I}$ is discrete. Since the restrictions of eulerian building sets are eulerian, the statement of theorem follows by induction of the rank of $\mathcal{B}$.

Let $\bar{\alpha} \models n$ be the opposite composition of a composition $\alpha \models n$, defined by $S(\bar{\alpha})=$ $[n-1] \backslash S(\alpha)$. It is an immediate corollary of Theorem 7.1 that the flag $h$-vector of an eulerian building set is symmetric

$$
\eta_{\alpha}(\mathcal{B})=\eta_{\bar{\alpha}}(\mathcal{B}), \text { for all } \alpha=n \text { and } \mathcal{B} \in \mathcal{E S e t}_{n} .
$$

This is equivalent to $\Psi_{\mathcal{B}}(\mathbf{a}, \mathbf{b})=\Psi_{\mathcal{B}}(\mathbf{b}, \mathbf{a})$, which is a consequence of $\Psi_{\mathcal{B}}(\mathbf{a}, \mathbf{b})=\Phi_{\mathcal{B}}(\mathbf{a}+$ $\mathbf{b}, \mathbf{a b}+\mathbf{b a})$.

For $j \geqslant 1$ define $\omega(j)=\left\{\begin{array}{cl}\frac{1}{2}\left(\mathbf{c}^{2}-2 \mathbf{d}\right)^{\frac{j-1}{2}} \mathbf{c}, & j \text { odd } \\ -\frac{1}{2}\left(\mathbf{c}^{2}-2 \mathbf{d}\right)^{\frac{j}{2}}, & j \text { even }\end{array}\right.$ and $\delta_{j}=\left\{\begin{array}{cc}0, & j \text { even } \\ \left(c^{2}-2 d\right)^{\frac{j-1}{2}}, & j \text { odd }\end{array}\right.$. By iterating the recursive formula (16) we obtain more explicitly 


$$
\Phi_{\mathcal{B}}(\mathbf{c}, \mathbf{d})=\sum_{k \geqslant 1} \sum_{\alpha=\left(a_{1}, \ldots, a_{k}\right) \models n} \zeta_{\alpha}(\mathcal{B}) \omega\left(a_{1}\right) \cdots \omega\left(a_{k-1}\right) \delta_{a_{k}}, \mathcal{B} \in \mathcal{E S e t}_{n}
$$

Example 7.2. Let $\Phi_{n}=\Phi_{\mathcal{D}_{n}}(\mathbf{c}, \mathbf{d})$ be the $\mathbf{c d}$-index of the discrete building set $\mathcal{D}_{n}$. From the recursive formula (16) we obtain

$$
\Phi_{n}=\frac{1}{2} \sum_{k \text { odd }}\left(\begin{array}{l}
n \\
k
\end{array}\right)\left(\mathbf{c}^{2}-2 \mathbf{d}\right)^{\frac{k-1}{2}} \mathbf{c} \Phi_{n-k}-\frac{1}{2} \sum_{k \text { even }}\left(\begin{array}{l}
n \\
k
\end{array}\right)\left(\mathbf{c}^{2}-2 \mathbf{d}\right)^{\frac{k}{2}} \Phi_{n-k}+\delta_{n}
$$

For instance,

$$
\Phi_{2}=\mathbf{c}, \Phi_{3}=\mathbf{c}^{2}+\mathbf{d}, \Phi_{4}=\mathbf{c}^{3}+2(\mathbf{c d}+\mathbf{d} \mathbf{c}), \Phi_{5}=\mathbf{c}^{4}+3\left(\mathbf{c}^{2} \mathbf{d}+\mathbf{d} \mathbf{c}^{2}\right)+5 \mathbf{c d} \mathbf{c}+4 \mathbf{d}^{2} .
$$

The following numerical identity is obtained from (16) and (17) by calculating the coefficient by $\mathbf{c}^{n-1}$ in $\Phi_{n}$

$$
\left[\mathbf{c}^{n-1}\right] \Phi_{n}=1=\sum_{\substack{\alpha \models n, a_{k(\alpha)} \text { odd }}}\left(\begin{array}{l}
n \\
\alpha
\end{array}\right) \frac{(-1)^{e(\alpha)}}{2^{k(\alpha)-1}} .
$$

By compering (18) with the similar recurrence relation satisfied by the $\mathbf{c d}-\operatorname{index} U_{B_{n}}(\mathbf{c}, \mathbf{d})$ of boolean posets $B_{n}$ ([16], Corollary 1.3), we obtain

$$
\Phi_{\mathcal{D}_{n}}(\mathbf{c}, \mathbf{d})=U_{B_{n}}(\mathbf{c}, \mathbf{d}),
$$

which is recognized as the certain combinatorially defined polynomial, called Andre polynomial. The generating function of $\Phi_{n}([16]$, Corollary 1.4) is given by

$$
\sum_{n \geqslant 1} \Phi_{n} \frac{x^{n}}{n !}=\frac{2 \sinh (\mathbf{a}-\mathbf{b}) x}{\mathbf{a}-\mathbf{b}}\left(1-\frac{\mathbf{c} \sinh (\mathbf{a}-\mathbf{b}) x}{\mathbf{a}-\mathbf{b}}+\cosh (\mathbf{a}-\mathbf{b}) x\right)^{-1} .
$$

\section{The geometric characterization of eulerian building sets}

In this section we give the complete characterization of the class of eulerian building sets by some geometric conditions. To an antichain $\mathcal{L}$ of subsets of the ground set $X$ is associated its nerve $\Delta(\mathcal{L})$, which is an abstract simplicial complex on the vertex set $\mathcal{L}$, defined by

$$
\Delta(\mathcal{L})=\{\mathcal{S} \subset \mathcal{L} \mid \cap \mathcal{S} \neq \emptyset\}
$$

Let $|\Delta(\mathcal{L})|$ be the geometric realization of the nerve $\Delta(\mathcal{L})$ and $\operatorname{link}_{\Delta(\mathcal{L})}(\{S\})=\{\mathcal{S} \in$ $\Delta(\mathcal{L}) \mid S \notin \mathcal{S},(\cap \mathcal{S}) \cap S \neq \emptyset\}$ and $\operatorname{star}_{\Delta(\mathcal{L})}(\{S\})=\{\mathcal{S} \in \Delta(\mathcal{L}) \mid S \in \mathcal{S}\}$ be the link and the star of a vertex $\{S\}$ in the complex $\Delta(\mathcal{L})$. 
Definition 8.1. Let $e_{\mathcal{L}}: \Delta(\mathcal{L}) \rightarrow \mathbb{N}$ be an assignment $e_{\mathcal{L}}(\mathcal{S})=|\cap \mathcal{S}|$ of the number of elements in the intersection $\cap \mathcal{S}$ to a simplex $\mathcal{S} \in \Delta(\mathcal{L})$. The collection $\mathcal{L}$ is odd if $e_{\mathcal{L}}(\mathcal{S})$ is odd for all $\mathcal{S} \in \Delta(\mathcal{L})$. A simplex $\mathcal{S} \in \Delta(\mathcal{L})$ is close to a vertex $S \in \mathcal{L}$ if $S^{\prime} \cap S \neq \emptyset$ for all $S^{\prime} \in \mathcal{S}$. A simplex $\mathcal{S}$ is far from a vertex $S$ if it is not close to $S$.

The following lemma is clear from definitions.

Lemma 8.2. Given an element $S \in \mathcal{L}$ of an antichain $\mathcal{L}$, then

$$
\begin{gathered}
\Delta(\mathcal{L} \backslash\{S\})=\Delta(\mathcal{L}) \backslash \operatorname{star}_{\Delta(\mathcal{L})}(\{S\}), \\
e_{\mathcal{L} \backslash\{S\}}(\mathcal{S})=e_{\mathcal{L}}(\mathcal{S}), \text { for all } \mathcal{S} \in \Delta(\mathcal{L} \backslash\{S\}), \\
\Delta(\mathcal{L} / S)=\Delta(\mathcal{L} \backslash\{S\}) \cup \mathcal{P}\left(\left\{S^{\prime} \in \mathcal{L} \mid S^{\prime} \cap S \neq \emptyset\right\}\right), \\
e_{\mathcal{L} / S}(\mathcal{S} / S)=\left\{\begin{array}{cc}
e_{\mathcal{L}}(\mathcal{S}), & \text { if } \mathcal{S} \text { is far from } S \\
e_{\mathcal{L}}(\mathcal{S})-e_{\mathcal{L}}(\mathcal{S} \cup\{S\})+1, & \text { if } \mathcal{S} \text { is close to } S
\end{array} .\right.
\end{gathered}
$$

Definition 8.3. An antichain $\mathcal{L}=\left\{S_{1}, \ldots, S_{k}\right\}$ of subsets of $X$ is a $k$-clique if $\cap \mathcal{L} \neq \emptyset$, i.e. the nerve $\Delta(\mathcal{L})$ is a simplex on the vertex set $\mathcal{L}$.

Lemma 8.4. Let $\mathcal{L}=\left\{S_{1}, \ldots, S_{k}\right\}$ be a $k$-clique on $X$. The building set $\mathcal{B}(\mathcal{L})$ is eulerian if and only if $\mathcal{L}$ is an odd collection.

Proof. We prove the statement by induction on $k$. For $k=1$, we have $\mathcal{B}(\{S\})=\overline{\mathcal{D}}_{|S|}$. Hence, by example $6.8, \mathcal{B}(\{S\})$ is eulerian if and only if $|S|$ is odd. Suppose the statement is true for any $(k-1)$-clique. Let a $k$-clique $\mathcal{L}=\left\{S_{1}, \ldots, S_{k}\right\}$ be an odd collection and $\mathcal{B}=\mathcal{B}(\mathcal{L})$. By the deletion-contraction property we have

$$
\zeta^{-1}(\mathcal{B})=\zeta^{-1}\left(\mathcal{B} \backslash S_{k}\right)-\zeta^{-1}\left(\mathcal{B} / S_{k}\right) .
$$

By Lemma 8.2, collections $\mathcal{L} \backslash\left\{S_{k}\right\}$ and $\mathcal{L} / S_{k}$ are odd $(k-1)$-cliques, so $\zeta^{-1}(\mathcal{B})=0$ by induction. For an arbitrary proper subset $J \subset X$ let $I=\left\{i \in[k] \mid S_{i} \subset J\right\}, X_{I}=\cup_{i \in I} S_{i}$ and $\mathcal{L}_{I}=\left\{S_{i} \mid i \in I\right\}$. We have

$$
\left.\mathcal{B}\right|_{J}=\mathcal{B}\left(\mathcal{L}_{I}\right) \sqcup \mathcal{D}_{|J|-\left|X_{I}\right|} .
$$

The collections $\mathcal{L}_{I}, I \subset[k]$ are odd cliques, so $\mathcal{B}$ is eulerian by induction.

Suppose that $\mathcal{L}=\left\{S_{1}, \ldots, S_{k}\right\}$ is a $k$-clique such that $e_{\mathcal{L}}(\mathcal{S})$ is odd for all proper subcollections $\mathcal{S} \subset \mathcal{L}$ and $e_{\mathcal{L}}(\mathcal{L})$ is even. We say that such collection is almost odd. The collection $\mathcal{L} \backslash\left\{S_{k}\right\}$ is an odd $(k-1)$-clique. Hence by the deletion-contraction property we have

$$
\zeta^{-1}(\mathcal{B}(\mathcal{L}))=-\zeta^{-1}\left(\mathcal{B}(\mathcal{L}) / S_{k}\right)
$$


By Lemma 8.2, the collection $\mathcal{L} / S_{k}$ is almost odd. We obtain by induction on $k$ that $\zeta^{-1}(\mathcal{B}(\mathcal{L}))=(-1)^{k-1} \zeta^{-1}\left(\overline{\mathcal{D}}_{d}\right)$, for some even $d$. Therefore $\zeta^{-1}(\mathcal{B}(\mathcal{L}))=(-1)^{k-1} 2$ and $\mathcal{B}(\mathcal{L})$ is not eulerian.

Let $\mathcal{L}=\left\{S_{1}, \ldots, S_{k}\right\}$ be a $k$-clique which is neither odd nor almost odd and $\mathcal{S} \subset \mathcal{L}$ be a minimal almost odd subcollection. The building set $\mathcal{B}(\mathcal{S})$ is a restriction of $\mathcal{B}(\mathcal{L})$ such that $\zeta^{-1}(\mathcal{B}(\mathcal{S})) \neq 0$. Consequently, $\mathcal{B}(\mathcal{L})$ is not eulerian.

A simplicial complex $\Delta$ on the set of vertices $V$ is a flag complex if for an arbitrary subset of vertices $S \subset V$, such that $\{i, j\} \in \Delta$ for all $i, j \in S$, it follows that $S \in \Delta$.

Proposition 8.5. Let $\mathcal{L}$ be an antichain of subsets of the ground set $X$, such that the corresponding building set $\mathcal{B}(\mathcal{L})$ is eulerian. Then $\mathcal{L}$ is odd and the nerve $\Delta(\mathcal{L})$ is a flag complex.

Proof. Let $\mathcal{S} \in \Delta(\mathcal{L})$ be a clique. The building set $\mathcal{B}(\mathcal{S})$ is eulerian as a restriction of $\mathcal{B}(\mathcal{L})$. Hence, by Lemma $8.4, \mathcal{S}$ is an odd collection. Thus $e_{\mathcal{L}}(\mathcal{S})$ is odd for every $\mathcal{S} \in \Delta(\mathcal{L})$, so the collection $\mathcal{L}$ is odd.

We have to prove that all minimal non-simplices of the complex $\Delta(\mathcal{L})$ are edges. Suppose that there is a collection $\mathcal{S}=\left\{S_{1}, \ldots, S_{k}\right\} \subset \mathcal{L}$, where $k>2$, which is a minimal non-simplex of $\Delta(\mathcal{L})$. It means that $\cap \mathcal{S}=\emptyset$ and $\cap \mathcal{S}_{i} \neq \emptyset$ for all subcollections $\mathcal{S}_{i}=\mathcal{S} \backslash\left\{S_{i}\right\}, i \in[k]$. Since $\zeta^{-1}\left(\mathcal{B}\left(\mathcal{S}_{k}\right)\right)=0$, it follows from the deletion-contraction property that

$$
\zeta^{-1}(\mathcal{B}(\mathcal{S}))=-\zeta^{-1}\left(\mathcal{B}(\mathcal{S}) / S_{k}\right)
$$

By Lemma 8.2, we obtain $e_{\mathcal{S} / S_{k}}\left(\mathcal{S}_{k} / S_{k}\right)=e_{\mathcal{S}}\left(\mathcal{S}_{k}\right)+1$, which is even, and $e_{\mathcal{S} / S_{k}}\left(\mathcal{S}^{\prime} / S_{k}\right)=$ $e_{\mathcal{S}}\left(\mathcal{S}^{\prime}\right)-e_{\mathcal{S}}\left(\mathcal{S}^{\prime} \cup\left\{S_{k}\right\}\right)+1$, for all $\mathcal{S}^{\prime} \subset \mathcal{S}_{k}$, which are odd. Hence the clique $\mathcal{S} / S_{k}$ is almost odd. From the proof of Lemma 8.4, we have $\zeta^{-1}\left(\mathcal{B}(\mathcal{S}) / S_{k}\right)=(-1)^{k-2} 2$ and consequently $\zeta^{-1}(\mathcal{B}(S))=(-1)^{k-1} 2$, which contradicts the condition that $\mathcal{B}(\mathcal{L})$ is eulerian.

To an antichain $\mathcal{L}=\left\{S_{1}, \ldots, S_{m}\right\}$ is associated the intersection graph $\Gamma(\mathcal{L})$, with the set of vertices $V(\Gamma(\mathcal{L}))=\mathcal{L}$ and the set of edges $E(\Gamma(\mathcal{L}))=\left\{\left\{S_{i}, S_{j}\right\} \mid S_{i} \neq S_{j}, S_{i} \cap S_{j} \neq \emptyset\right\}$, which is a simple graph. To a simple graph $\Gamma=(V, E)$ we associate an abstract simplicial complex $\Delta(\Gamma)$, called a clique complex. A subset of vertices $S \subset V$ is a clique of $\Gamma$ if $\{i, j\} \in$ $E$ for all $i, j \in S$. The clique complex is defined by $\Delta(\Gamma)=\{S \subset V \mid S$ is a clique of $\Gamma\}$. We have that a flag complex is a clique complex of its 1 -skeleton. By Proposition 8.5, for eulerian building set $\mathcal{B}(\mathcal{L})$, the nerve $\Delta(\mathcal{L})$ of an antichain $\mathcal{L}$ is a clique complex $\Delta(\Gamma(\mathcal{L}))$ of the intersection graph $\Gamma(\mathcal{L})$.

Lemma 8.6. Let $\mathcal{L}$ be an odd antichain of finite sets such that the nerve $\Delta(\mathcal{L})$ is a 1 -dimensional cycle on $n=|\mathcal{L}|$ vertices. Then $\zeta^{-1}(\mathcal{B}(\mathcal{L}))=2(-1)^{n-1}$.

Proof. By Proposition 4.9, we have $\zeta^{-1}(\mathcal{B}(\mathcal{L}))=\zeta^{-1}\left(\beta_{3}\left(C_{n}\right)\right)$, where $C_{n}$ is the cycle graph on $n$ vertices. It follows from the deletion-contraction property that 


$$
\zeta^{-1}\left(\beta_{3}\left(C_{n}\right)\right)=-\zeta^{-1}\left(\beta_{3}\left(L_{n}\right)\right)-\zeta^{-1}\left(\beta_{3}\left(C_{n-1}\right)\right)
$$

where $L_{n}$ is the path on $n$ vertices. Since $\zeta^{-1}\left(\beta_{3}\left(L_{n}\right)\right)=0$ by Lemma 6.5, we obtain $\zeta^{-1}\left(\beta_{3}\left(C_{n}\right)\right)=(-1)^{n-3} \zeta^{-1}\left(\beta_{3}\left(C_{3}\right)\right)$ by induction on $n$. The direct calculation gives $\zeta^{-1}\left(\beta_{3}\left(C_{3}\right)\right)=2$.

Let $\Delta$ be an abstract simplicial complex on the vertex set $V$. A restriction $\left.\Delta\right|_{I}$ of the complex $\Delta$ to a subset of vertices $I \subset V$ is defined by $\left.\Delta\right|_{I}=\{\sigma \in \Delta \mid \sigma \subset I\}$. A subcomplex of the form $\left.\Delta\right|_{I}$ is called full subcomplex.

Definition 8.7. We say that an abstract simplicial complex $\Delta$ is fully acyclic if it does not contain any full subcomplex which is a 1-dimensional cycle.

Note that the 1-skeleton of a fully acyclic simplicial complex is a chordal graph. Recall that a graph $\Gamma$ is called chordal if for each of its cycles on more than three vertices there is an edge joining two vertices that are not adjacent in the cycle.

Proposition 8.8. Let $\mathcal{L}$ be an antichain of finite sets such that the building set $\mathcal{B}(\mathcal{L})$ is eulerian. Then the nerve $\Delta(\mathcal{L})$ is a fully acyclic complex.

Proof. Suppose that there is a subcollection $\mathcal{S} \subset \mathcal{L}$ such that the nerve $\Delta(\mathcal{S})$ is a full subcomplex of $\Delta(\mathcal{L})$ which is a 1-dimensional cycle. By Proposition 8.5, $\mathcal{S}$ is odd as a subcollection of the odd collection $\mathcal{L}$. By Lemma 8.6, we have $\zeta^{-1}(\mathcal{B}(\mathcal{S})) \neq 0$, which contradicts the condition that $\mathcal{B}(\mathcal{L})$ is eulerian.

Remark 8.9. A flag simplicial complex is fully acyclic if and only if its 1-skeleton is chordal. The class of fully acyclic flag complexes is closed under taking restrictions of a simplicial complex.

Proposition 8.10. Let $\mathcal{L}$ be an odd antichain of finite sets such that the nerve $\Delta(\mathcal{L})$ is a fully acyclic flag complex. Then, the building set $\mathcal{B}(\mathcal{L})$ is eulerian.

Proof. We prove the statement by induction on the number of elements $n=|\mathcal{L}|$ of an antichain $\mathcal{L}$. The statement is true for $n=2$ by direct consideration. Suppose that the statement is true for any antichain with at most $n-1$ elements. Let $\mathcal{L}$ be a connected odd antichain of $n$ finite sets, such that the nerve $\Delta(\mathcal{L})$ is a fully acyclic flag complex. The restrictions $\Delta(\mathcal{S})$ are fully acyclic flag complexes for all subcollection $\mathcal{S} \subset \mathcal{L}$. Hence, by induction, we only need to prove that $\zeta^{-1}(\mathcal{B}(\mathcal{L}))=0$. Let $S_{0} \in \mathcal{L}$ be an arbitrary element. Since $\zeta^{-1}\left(\mathcal{B}(\mathcal{L}) \backslash S_{0}\right)=0$ by induction, we obtain by the deletion-contraction property

$$
\zeta^{-1}(\mathcal{B}(\mathcal{L}))=-\zeta^{-1}\left(\mathcal{B}(\mathcal{L}) / S_{0}\right)
$$

Let $\mathcal{S}_{0}=\left\{S \in \mathcal{L} \backslash\left\{S_{0}\right\} \mid S \cap S_{0} \neq \emptyset\right\}$ be the collection of close vertices to the vertex $S_{0}$, $\sigma=\left|\mathcal{P}\left(\mathcal{S}_{0}\right)\right|$ be the geometrical simplex on vertices $\mathcal{S}_{0}$ and $\Delta\left(\mathcal{S}_{0}\right)$ be the nerve of the 
collection $\mathcal{S}_{0}$. By Lemma 8.2, the geometric realization of the nerve $\Delta\left(\mathcal{L} / S_{0}\right)$ is obtained as

$$
\left|\Delta\left(\mathcal{L} / S_{0}\right)\right|=\left|\Delta\left(\mathcal{L} \backslash\left\{S_{0}\right\}\right)\right| \cup_{\left|\Delta\left(\mathcal{S}_{0}\right)\right|} \sigma .
$$

The nerve $\Delta\left(\mathcal{S}_{0} \cup\left\{S_{0}\right\}\right)$ of the collection $\mathcal{S}_{0} \cup\left\{S_{0}\right\}$ is a flag complex as a full subcomplex of the flag complex $\Delta(\mathcal{L})$. Hence, by Lemma 8.2 and the fact that $\mathcal{L}$ is odd, we obtain that $e_{\mathcal{L} / S_{0}}\left(\mathcal{S} / S_{0}\right)$ is odd for all simpleces $\mathcal{S} / S_{0} \in \Delta\left(\mathcal{L} / S_{0}\right)$, where $\mathcal{S} \subset \mathcal{L} \backslash\left\{S_{0}\right\}$. If $\mathcal{S} / S_{0} \in \Delta\left(\mathcal{L} / S_{0}\right)$ is a collection which is not in $\Delta\left(\mathcal{L} \backslash\left\{S_{0}\right\}\right)$, we have $e_{\mathcal{L} / S_{0}}\left(\mathcal{S} / S_{0}\right)=1$. Thus, $\mathcal{L} / S_{0}$ is an odd collection.

Suppose that $\mathcal{S} \subset \mathcal{L}$ is a collection such that $\mathcal{S} / S_{0}$ is a minimal non-simplex of the nerve $\Delta\left(\mathcal{L} / S_{0}\right)$ with at least three vertices. The collection $\mathcal{S}$ is divided by $\mathcal{S}=\mathcal{S}_{c} \cup \mathcal{S}_{f}$, where $\mathcal{S}_{c}=\left\{S \in \mathcal{S} \mid S \cap S_{0} \neq \emptyset\right\}$ and $\mathcal{S}_{f}=\left\{S \in \mathcal{S} \mid S \cap S_{0}=\emptyset\right\}$. By the condition that $\mathcal{S} / S_{0}$ is a non-simplex, we have that $\mathcal{S}_{f} \neq \emptyset$ and $\left|\mathcal{S}_{c}\right| \geqslant 2$. We can find two vertices $S^{\prime}, S^{\prime \prime} \in \mathcal{S}_{c}$ such that $S^{\prime} \cap S^{\prime \prime}=\emptyset$ and a vertex $S \in \mathcal{S}_{f}$ such that $S \cap S^{\prime} \neq \emptyset$ and $S \cap S^{\prime \prime} \neq \emptyset$. Then $\left\{S_{0}, S, S^{\prime}, S^{\prime \prime}\right\}$ form a full cycle in $\Delta(\mathcal{L})$, contrary to the condition that $\Delta(\mathcal{L})$ is fully acyclic.

Suppose that there is a collection $\mathcal{S} \subset \mathcal{L} \backslash\left\{S_{0}\right\}$ such that $\Delta\left(\mathcal{S} / S_{0}\right)$ is a full subcomplex of $\Delta(\mathcal{L} / S)$ which is a 1-dimensional cycle. Then $\Delta\left(\mathcal{S} \cup\left\{S_{0}\right\}\right)$ is a 1-dimensional cycle which is a full subcomplex of $\Delta(\mathcal{L})$, contrary to the condition that $\Delta(\mathcal{L})$ is fully acyclic.

We obtain that $\Delta\left(\mathcal{L} / S_{0}\right)$ is fully acyclic flag complex. By induction, $\mathcal{B}(\mathcal{L}) / S_{0}$ is eulerian. Hence, $\zeta^{-1}\left(\mathcal{B}(\mathcal{L}) / S_{0}\right)=0$, which implies $\zeta^{-1}(\mathcal{B}(\mathcal{L}))=0$.

By Propositions 8.5, 8.8 and 8.10 and Remark 8.9 we obtain the complete characterization of eulerian building sets in terms of combinatorics of nerves of antichains of finite sets.

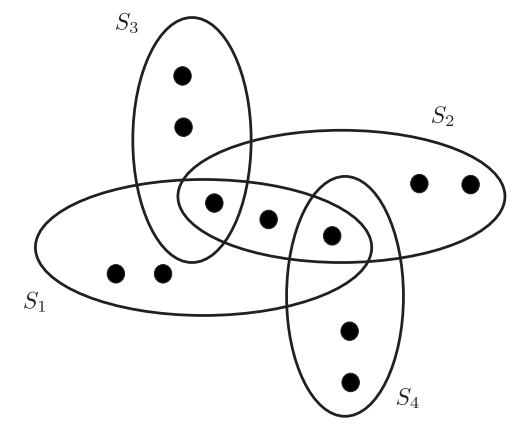

$\mathcal{B}=\mathcal{B}\left(\left\{S_{1}, S_{2}, S_{3}, S_{4}\right\}\right)$

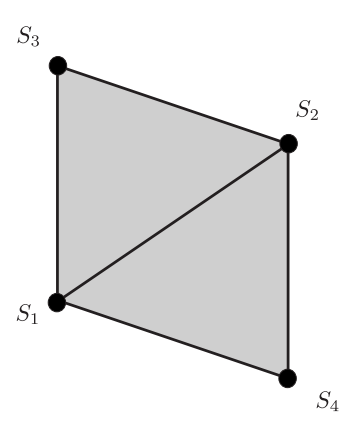

$\Delta\left(\left\{S_{1}, S_{2}, S_{3}, S_{4}\right\}\right)$

Figure 4: The eulerian building set $\mathcal{B} \in \mathcal{E}$ Set $_{11}$

Theorem 8.11. Let $\mathcal{L}$ be an antichain of finite sets. The building set $\mathcal{B}(\mathcal{L})$ is eulerian if and only if the collection $\mathcal{L}$ is odd and its nerve $\Delta(\mathcal{L})$ is the clique complex of a chordal graph. 
Example 8.12. A flag 1-dimensional fully acyclic complex is a tree. Let $\mathcal{L}$ be an antichain of finite sets such that the nerve $\Delta(\mathcal{L})$ is a tree. By Theorem $8.11, \mathcal{B}(\mathcal{L})$ is eulerian if and only if the collection $\mathcal{L}$ is odd.

Example 8.13. Let $\beta_{2 k+1}(\Gamma)$ be a building set formed by a simple graph $\Gamma=(V, E)$, for $k \geqslant 1$. The nerve of the collection $\left\{S_{e} \mid e \in E\right\}$ is the clique complex $\Delta\left(\Gamma^{*}\right)$ of the dual graph $\Gamma^{*}$, which is fully acyclic if and only if $\Gamma$ is a tree. Thus $\beta_{2 k+1}(\Gamma)$ is eulerian if and only if $\Gamma$ is a tree.

Remark 8.14. The building sets produce simple polytopes called nestohedra [8], [10]. The natural question to ask is how the property of being eulerian reflects on combinatorics of nestohedra produced from eulerian building sets.

\section{References}

[1] M. Aguiar. Infinitesimal Hopf algebras and the cd-index of polytopes. Discrete Comput. Geom. 27(1): 3-28, 2002.

[2] M. Aguiar, N. Bergeron and F. Sottile. Combinatorial Hopf algebras and generalized Dehn-Sommerville relations. Compositio Mathematica. 142: 1-30, 2006.

[3] M. Bayer and L. Billera. Generalized Dehn-Sommerville relations for polytopes, spheres, and Eulerian partially ordered sets. Invent. Math. 79: 143-157, 1985.

[4] M. Bayer and A. Klapper. A new index for polytopes. Discrete Comput. Geom. 6: 33-47, 1991.

[5] T. Brylawski and J. Oxley. The Tutte polynomial and its applications. Matroid applications. Encyclopedia Math. Appl. Vol. 40, Cambridge Univ. Press, 1992.

[6] C. De Concini and C. Procesi. Wonderful models for subspace arrangements. Selecta Math. (N.S.) 1: 459-494, 1995.

[7] S. Dascalescu, C. Nastasescu and S. Raianu. Hopf algebras, An Introduction. Marcel Dekker, Inc. 2001.

[8] E. M. Feichtner and B. Sturmfels. Matroid polytopes, nested sets and Bergman fans. Port. Math. (N.S.) 62(4): 437-468, 2005.

[9] S. Joni and G.-C. Rota. Coalgebras and bialgebras in combinatorics. Stud. Appl. Math. 61: 93-139, 1979.

[10] A. Postnikov. Permutohedra, Associahedra, and Beyond. Int. Math. Res. Notices. 6: 1026-1106, 2009.

[11] R. Stanley. Enumerative combinatorics. Vol. 2. Cambridge Univ. Press, Cambridge, 1999.

[12] R. Stanley. A symmetric function generalization of the chromatic polynomial of a graph. Adv. in Math. 111: 166-194, 1995.

[13] R. Stanley. Graphs colorings and related symmetric functions: Ideas and Apllications. Discrete Math. 193: 267-286, 1998. 
[14] R. Stanley. Acyclic orientations of graphs. Discrete Math. 5: 171-178, 1973.

[15] R. Stanley. Combinatorial reciprocity theorems. Adv. in Math. 14: 194-253, 1974.

[16] R. Stanley. Flag $f$-vectors and the cd-index. Math. Z. 216: 483-499, 1994.

[17] W. R. Schmitt. Incidence Hopf algebras. J. Pure Appl. Algebra. 96: 299-330, 1994.

[18] W. R. Schmitt. Hopf Algebra Methods in Graph Theory. Journal of Pure and Applied Algebra. 101: 77-90, 1995.

[19] G. Whittle. A geometric theory of hypergraph coloring. Aequationes Math. 43: 45-58, 1992.

[20] H. Whitney. A logical expansion in mathematics. Bull. Amer. Math. Soc. 38: 572-579, 1932. 